\title{
Prevalence Of Glycaemic Variability (GV) And Factors Associated With The Glycaemic Arrays Among End-Stage Kidney Disease (ESKD) Patients On Chronic Haemodialysis.
}

\author{
Abdul Hanif Khan Yusof Khan \\ Universiti Putra Malaysia \\ Nor Fadhlina Zakaria ( $\nabla$ n_fadhlina@upm.edu.my) \\ Universiti Putra Malaysia https://orcid.org/0000-0002-2126-7391 \\ Muhammad Adil Zainal Abidin \\ International Islamic University Malaysia \\ Nor Azmi Kamaruddin \\ Universiti Kebangsaan Malaysia
}

Research article

Keywords: Glycaemic variability, diabetic nephropathy, risk factors, haemodialysis

Posted Date: July 14th, 2020

DOl: https://doi.org/10.21203/rs.3.rs-41271/v1

License: @ (1) This work is licensed under a Creative Commons Attribution 4.0 International License. Read Full License 


\section{Abstract}

Introduction: Glycaemic variability (GV) or glycaemic fluctuations carries a significant higher risk of diabetic-related complications, especially cardiovascular. Extensive researches have been reported, but study on the end-stage-kidney-disease (ESKD) patients on chronic haemodialysis are scarce. This study aims to determine the magnitude of GV among ESKD (diabetic vs non-diabetic) patients and its associated factors during haemodialysis day (HDD) and non-haemodialysis day (NHDD).

Methods: We recruited 150 patients on haemodialysis, 93 patients had diabetic (DM-ESKD), and 57 non-diabetic (NDM-ESKD). The GV indices (standard deviation [SD] and percentage co-efficient variant [\%CV]) were obtained from 11-point and 7-point selfmonitoring blood glucose (SMBG) profiles during haemodialysis and non-haemodialysis day. The GV indices and its associated factors were analysed comparing between both groups during the HDD and NHDD.

Results: Mean blood glucose during HDD was 9.33 [SD 2.7, \%CV 30.6\%] mmol/L in DM-ESKD compared to 6.07 [SD 0.85, \%CV 21.3\%] $\mathrm{mmol} / \mathrm{L}$ in NDM-ESKD $(p=<0.01)$. The DM-ESKD group experienced significantly higher GV indices compared to NDM-ESKD; both during haemodialysis and non-haemodialysis day, particularly in the group with $\mathrm{HbA} 1 \mathrm{c} 8-10 \%(p=<0.01)$. Presence of diabetes, older age group, hyperlipidaemia, $\mathrm{HbA1c}$, ferritin levels, and albumin were recognised as factors associated with GV.

Conclusion: DM-ESKD patients has high glycaemic variability, especially during the haemodialysis day, therefore increasing their chance to develop future devastating complications. We identified high $\mathrm{HbA} 1 \mathrm{c}$, older age group, presence of hyperlipidaemia, ferritin levels, and albumin as factors associated with GV. Since these groups of patients are vulnerable to CVD mortality, urgent attention is needed to rectify it.

\section{Background}

Type 2 Diabetes Mellitus (T2DM) is the primary cause of end-stage-kidney-disease (ESKD) worldwide [1, 2]. A similar trend was seen in Malaysia, where the prevalence rate had doubled over the last ten years, with almost two-thirds of patients has diabetes [3]. Diabetic ESKD (DM-ESKD) has higher morbidity and mortality, mainly related to cardiovascular complications with poor glycemic control proved to be a predictor of mortality [4]. Therefore, glycemic control had been a focus of extensive research, especially among DM-ESKD on haemodialysis, as they experienced more marked fluctuations in blood glucose compared to non-haemodialysis diabetic populations [5].

Glycemic variability (GV) had been coined to explain these glucose fluctuations among diabetic patients. GV is shown to be an independent risk factor for morbidity and mortality among the non-haemodialysis diabetic population as previous studies demonstrated targeting $\mathrm{HbA1c}$ alone, i.e., surrogate for chronic hyperglycemia, by intensive glucose-lowering treatment, had failed to show better cardiovascular outcomes [6-9].

Data from the general population had prompted concerns regarding glycemic control among ESKD patients that have higher cardiovascular risk. Large population studies among haemodialysis patients showed that there was an association between $\mathrm{HbA} 1 \mathrm{c}$ level value of less than $6 \%$ and more than $8 \%$ with decreased in overall survival [10,11]. This U-shape association in haemodialysis patients might indicate that chronic hyperglycemia per se is not an indicator of morbidity and mortality, but also hypoglycemia with glucose fluctuations that were more evident in these malnourished and protein-energy wasted patients $[5,11]$.

These findings indicate that reducing GV is an essential therapeutic option in reducing cardiovascular complications in the haemodialysis population. However, although GV are heavily investigated among non-ESKD diabetic patients, minimal data available in ESKD patients, especially on the extent of GV during haemodialysis and non-haemodialysis day. Furthermore, among Malaysian population, the data on GV is still lacking. We have conducted previous analysis on glycemic patterns during haemodialysis in our population that shows DM-ESKD experienced four times more pronounced post-haemodialysis hyperglycemia compared to their counterpart [12]. Therefore the objectives of this study are to determine further the magnitude and factors associated with GV among ESKD patient during haemodialysis (HDD) and non-haemodialysis day (NHDD) by utilizing self-monitoring-blood glucose (SMBG) measurement to optimize management in this group of population.

\section{Methods}




\section{Study Design}

In this cross-sectional study, we recruited 150 ESKD patients on maintenance haemodialysis with DM-ESKD $(n=93)$ and NDM-ESKD $(n=57)$. This study was approved by the ethical committee of University Putra Malaysia (UPM) and was conducted according to the Declaration of Helsinki, where written consent was obtained before the study. Patients were recruited from 5 private haemodialysis centres in Selangor, Malaysia. The sample size was calculated based on a study by Jin Y.P, 2015, which look at blood glucose fluctuations among haemodialysis population [13]. Multiple logistic regression using $G$ power software [14] was used by considering a model with one binary covariate $X$ with event rate under $H o, p_{1}=0.13$ and the event rate under $X=1, p_{2}=0.40$, giving the odds ratio of $\sim 4.5$. We further assumed $\mathrm{R}^{2}=0.1$, and an imbalanced design ratio of 2:1 between the two groups. The estimating sample size necessary to achieve a two-sided test with an alpha of 0.05 and power of at least $80 \%$ was 102 . The final sample size was 146 rounded to 150 , considering a $30 \%$ non-response rate.

The conditions for inclusion are as follows: adults over 18 years of age with or without diabetes, patients on maintenance haemodialysis for at least three months, patients with stable haemoglobin levels over the last three months and patient with no recent change in insulin or oral hypoglycemic agents. Exclusion criteria were; Type 1 diabetes mellitus patients, history of blood transfusion or hospitalization for the previous three months, hemoglobinopathy, presence of the acute inflammatory state, and diagnosis of malignancy.

\section{Socio-demographic And Comorbidities Data}

A structured questionnaire has been developed for socio-demographic information, medical information, comorbidities and prescription lists. Baseline blood tests were taken from patients at the start of the study. All blood taken was sent to Prima Lab Sdn. Bhd., Puchong, Selangor.

\section{Self-monitor-blood-glucose (smbg)}

Capillary glucose measurement was measured using capillary glucometers (Bayer contour plus $®$ ) in this study. All patients were trained to measure capillary glucose and to record glucose values. Patients were also taught to recognize symptoms of hypoglycemia and to record the blood glucose during the events.

During the haemodialysis day, an 11-point capillary self-monitoring glucose (SMBG) profile was obtained as follows, i.e. fasting, prehaemodialysis, hourly during haemodialysis, followed by pre-and post-meal glucose readings at home. During haemodialysis hours, patients were assisted in the measurement of blood glucose levels. During the non-haemodialysis day, a 7-point SMBG profile was measured during fasting, pre-and post-meal during breakfast, lunch and dinner. Concerning medications, patients were advised to continue taking their current drugs as usual, either during haemodialysis or non-haemodialysis days. Patients were not required to fast during the duration of haemodialysis. Patients have been advised to eat as usual and log whenever they take food out of the ordinary.

\section{Assessment Of Glycemic Variability (gv)}

We choose standard deviation (SD) and percentage co-efficient (\%CV) as indices of GV that were calculated from 11-points SMBG and 7-point SMBG during haemodialysis and non-haemodialysis day. The SD was calculated as arithmetic SD, and \%CV is obtained by ([SD of glucose]/[mean glucose]) $\times 100$. In terms of target GV, the ideal target for SD calculated was from the following formula, i.e., $\mathrm{SD} \times 3<$ mean glucose and for \%CV the value of $<36 \%[15,16]$.

\section{Statistical analysis}

The analysis was performed using RStudio. The GV was calculated using two methods, i.e. SD and \%CV. The data were checked for normality visually using a histogram and statistically using the Shapiro Wilk test. For univariate analysis, chi-square test and independent-sample t-test were used. The assumption for equal variance was met using Levene's test. All test was two-sided, and the level of significance was set at 0.05 . The association between GV with clinical and laboratory result were determined using simple 
logistic regression to derived crude odd ratio. We calculate each method of GV for haemodialysis day and non-haemodialysis day. Subsequently, the variables which were significant at $p<0.15$ were included in the final multivariable logistic regression analysis. All the crude and adjusted odds ratios were presented with $95 \%$ confidence intervals. For missing data, the listwise deletion method was used.

\section{Results}

\section{Baseline Socio-demographic, Clinical Characteristics And Blood Parameters Of Patients}

Summary of baseline socio-demographic, clinical characteristics and blood parameters of patients were described previously [12]. A total of 148 patients were involved in the final analysis after excluding missing data. DM-ESKD accounted for 91 (61.5\%) of patients with a mean age of 57.6 years and mean duration of diabetes of 16.4 years. The mean duration of haemodialysis between DM-ESKD and NDM-ESKD was 3.8 and 4.5 years, respectively, and not statistically significant. The difference in the prevalence of cardiovascular-related illness, e.g., hypertension, ischemic heart disease (IHD), hyperlipidemia, stroke, and gout in both groups, was not statistically significant; however, a quarter of patients reported to have previously documented IHD.

Blood pressure control group in both groups were suboptimal with only $16(10.7 \%)$ of DM-ESKD and 47 (31.3\%) of NDM-ESKD patients achieving pre and post haemodialysis target blood pressure of less or equal to $130 / 80 \mathrm{mmHg}$. Majority of DM-ESKD, 50 (54.9\%) patients were on insulin therapy with a quarter of patients not on any treatment. Medication intake during haemodialysis day depending on the type of treatment where patients on OHA alone would not take their OHA on haemodialysis days, while patient on basal-bolus insulin, would omit the insulin dose before their haemodialysis session. However, the majority of patients, i.e. 56 (82.3\%) patients, would not take their medications on haemodialysis days.

In general, both groups had a statistically non-significant difference in terms of blood parameters apart from HbA1c, phosphate, and albumin. The mean $\mathrm{HbA} 1 \mathrm{c}$ among DM-ESKD patients was $7.4 \%$, with around one-third having $\mathrm{HbA} 1 \mathrm{c}$ less than $6.5 \%$ while another $30 \%$ of patients documented $\mathrm{HbA} 1 \mathrm{c}$ in the range of $6.5-8 \%$. Albumin was lower in the DM-ESKD group, while phosphate is higher in NDM-ESKD. Highly sensitive C-reactive protein (hs-CRP) was used as a surrogate marker for cardiovascular risk, and both groups had a high hs-CRP level with a mean of $8.91 \mathrm{mg} / \mathrm{L}$ in and 7.03 in DM-ESKD and NDM-ESKD respectively.

\section{Gv Among Eskd Patients}

\section{GV during haemodialysis day}

Mean blood glucose \pm SD during haemodialysis among DM-ESKD and NDM-ESKD in our study was $9.33 \pm 2.7 \mathrm{mmol} / \mathrm{L}$ vs $6.07 \pm$ $0.85 \mathrm{mmol}$, respectively. Table 1 comparing GV indices between DM-ESKD and NDM-ESKD patients during haemodialysis day. Majority of our patients achieved target GV i.e. 111 (75.0\%), based on SD and 121 (81.8\%) based on \%CV with higher mean GV for DM-ESKD [SD: 2.7; \%CV: 30.6\%] compared to NDM-ESKD [SD: 0.85, \%CoV: 21.3\%; p 0.01]. DM-ESKD had higher prevalence of high GV compared to NDM-ESKD [SD: $33.0 \%$, $\%$ CV: $25.3 \%$ vs. SD: $12.2 \%, \% \mathrm{CV}: 7.1 \%$ ). In terms of HbA1c, GV indices were the highest in Hba1c group $8-10 \%$ while the lowest in the $<6.5 \%$ during haemodialysis day. Figure 1 represents the glycemic pattern based on GV indices on haemodialysis day.

Table 1:

Comparison of GV indices between DM-ESKD and NDM-ESKD during haemodialysis and non-haemodialysis day. 


\begin{tabular}{|c|c|c|c|c|c|}
\hline GV indices & $\begin{array}{c}\text { All patients, } \mathrm{n}(\%) \\
\mathrm{n}=148\end{array}$ & $\begin{array}{c}\text { DM-ESKD, n (\%) } \\
\mathrm{n}=91\end{array}$ & $\begin{array}{c}\text { NDM-ESKD, n (\%) } \\
n=57\end{array}$ & t statistic & p-value \\
\hline \multicolumn{6}{|l|}{ HD } \\
\hline $\begin{array}{l}\text { SD } \\
\text { Target GV: }(<\text { mean/3) } \\
\text { High GV: }(>\text { mean/3) }\end{array}$ & $\begin{array}{l}111(75.0) \\
37(25.0)\end{array}$ & $\begin{array}{l}61(67.0) \\
30(33.0)\end{array}$ & $\begin{array}{l}50(87.7) \\
7(12.2)\end{array}$ & 8.700 & $0.003^{*}$ \\
\hline $\begin{array}{l}\% \text { CV } \\
\text { Target GV: <36\% } \\
\text { High GV: > 36\% }\end{array}$ & $\begin{array}{l}121(81.8) \\
27(18.2)\end{array}$ & $\begin{array}{l}68(74.7) \\
23(25.3)\end{array}$ & $\begin{array}{c}53(92.9) \\
4(7.1)\end{array}$ & 8.726 & $0.003^{*}$ \\
\hline \multicolumn{6}{|l|}{ NHD } \\
\hline $\begin{array}{l}\text { SD } \\
\text { Target GV: }(<\text { mean/3) } \\
\text { High GV: }(>\text { mean/3) }\end{array}$ & $\begin{aligned} 137 & (92.6) \\
11 & (7.4)\end{aligned}$ & $\begin{array}{l}80(87.9) \\
11(12.1)\end{array}$ & $\begin{array}{c}57(100) \\
0(0)\end{array}$ & 6.649 & $0.009 *$ \\
\hline $\begin{array}{l}\% \text { CV } \\
\text { Target GV: <36\% } \\
\text { High GV: > 36\% }\end{array}$ & $\begin{array}{c}139(93.9) \\
8(6.1)\end{array}$ & $\begin{array}{c}83(91.2) \\
8(8.8)\end{array}$ & $\begin{array}{c}57(100) \\
0(0)\end{array}$ & 4.545 & $0.033^{*}$ \\
\hline
\end{tabular}

Glycaemic variability (GV) indices (SD, standard deviation; \%CV, percentage co-efficient variant) among all patients and also comparing DMESKD and NDM-ESKD during haemodialysis day (HD) and non-haemodialysis day (NHD) (student t-test). The results showed in the number (n) and percentage. $* \mathrm{p}<0.05$.

\section{Gv During Non-haemodialysis Day}

During non-haemodialysis day, mean blood glucose \pm SD among DM-ESKD and NDM-ESKD was $9.85 \pm 3.1 \mathrm{mmol} / \mathrm{L}$ vs $6.0 \pm$ $0.88 \mathrm{mmol}$, respectively. Mean GV for DM-ESKD was higher [SD: 3.1; \%CV: $22.9 \%$ ] compared to NDM-ESKD [SD: 0.88, \%CV: 15.1\%; $<$ < 0.01] with $12.1 \%(\mathrm{SD})$ and $8.8 \%(\% \mathrm{CV})$ of DM-ESKD experienced high GV. None of the NDM-ESKD patients demonstrated high GV indices during non-haemodialysis day. In terms of $\mathrm{HbA} 1 \mathrm{c}$, better $\mathrm{GV}$ target in the $\mathrm{HbA} 1 \mathrm{c}<6.5 \%$ group, while $8-10 \% \mathrm{HbA} 1 \mathrm{c}$ group demonstrated the highest GV indices. Figure 1 represents the glycemic pattern based on GV indices on non-haemodialysis day.

\section{Gv Haemodialysis And Non-haemodialysis Day}

In general, statistically significantly more patients achieved target GV during non-haemodialysis day as compared to haemodialysis day (SD: $75.0 \%$ vs $92.6 \%$; CV: $82.0 \%$ vs $92.7 \%$ ). However, when we factor in diabetic status, the results were not statistically significant (Table 2).

Table 2

Comparison of GV indices between haemodialysis and non-haemodialysis day

\begin{tabular}{|c|c|c|c|c|}
\hline \multirow[t]{2}{*}{ SD } & \multicolumn{2}{|c|}{ Proportion of target } & \multirow[t]{2}{*}{ c statistic } & \multirow[t]{2}{*}{ p-value } \\
\hline & HD, n (\%) & NHD, n (\%) & & \\
\hline All Patients & $111(75.0)$ & 137 (92.6) & 16.499 & $<0.001 *$ \\
\hline DM-ESKD & $61(67.7)$ & $80(56.3)$ & 0.25 & 0.6171 \\
\hline NDM-ESKD & $50(46.8)$ & $57(53.2)$ & & \\
\hline \multirow[t]{2}{*}{$\% \mathrm{CV}$} & \multicolumn{2}{|c|}{ Proportion of target } & c statistic & p-value \\
\hline & $\mathrm{HD}, \mathrm{n}(\%)$ & NHD, n (\%) & & \\
\hline All Patients & 121 (82.0) & 137 (92.7) & 11.316 & $<0.001 *$ \\
\hline DM-ESKD & $68(41.4)$ & $83(58.6)$ & 1.132 & 0.2874 \\
\hline NDM-ESKD & $53(48.2)$ & $57(51.8)$ & & \\
\hline
\end{tabular}


Glycaemic variability (GV) indices (SD, standard deviation; \%CV, percentage co-efficient variant) among all patients and also comparing haemodialysis (HD) and non-haemodialysis day (NHD) (chi-square analysis). The result is shown in number (n) and percentage. $*$ p $<0.05$. DM-ESKD, diabetic-end stage renal disease; NDM-ESKD, non-diabetic end-stage-renal-disease.

\section{Factors Associated With High Gv Among Eskd Patients}

Tables 3 and 4 demonstrated the association between clinical characteristics and blood parameters with GV on haemodialysis and non-haemodialysis day. In this study, the presence of diabetes and older age group was associated with high GV. While an additional presence of hyperlipidemia denotes higher GV during non-haemodialysis day. In terms of blood parameters, HbA1c, ferritin, LDL and TG associated with high GV during haemodialysis day. During non-haemodialysis day HbA1c, albumin and ferritin associated with high GV. Further multivariate analysis (Tables 5 and 6) showed that age and LDL were factors associated with GV during haemodialysis while albumin during non-haemodialysis day. In our study, we found no association between comorbidities and type of medications with GV. 
Table 3

is a simple logistic regression analysis of socio-demographic, clinical comorbidities and blood parameters among patients in cohort $(n=150)$ with poor GV during HD.

\begin{tabular}{|c|c|c|c|c|c|c|c|c|c|c|}
\hline & \multicolumn{2}{|l|}{ SD } & \multirow{2}{*}{$\begin{array}{l}\text { Test } \\
\text { statistic }\end{array}$} & \multirow{2}{*}{$\begin{array}{l}\text { p- } \\
\text { value }\end{array}$} & \multirow{2}{*}{$\begin{array}{l}\text { Crude Odds } \\
\text { ratio }(95 \% \\
\text { Cl) }\end{array}$} & \multicolumn{2}{|l|}{$\% \mathrm{CV}$} & \multirow{2}{*}{$\begin{array}{l}\text { Test } \\
\text { statistic }\end{array}$} & \multirow{2}{*}{$\begin{array}{l}\text { p- } \\
\text { value }\end{array}$} & \multirow{2}{*}{$\begin{array}{l}\text { Crude } \\
\text { Odds ratio } \\
(95 \% \mathrm{Cl})\end{array}$} \\
\hline & Target & High & & & & Target & High & & & \\
\hline \multirow{2}{*}{$\begin{array}{l}\text { Diabetes } \\
\text { No } \\
\text { Yes }\end{array}$} & $\begin{array}{l}50 \\
(45.0)\end{array}$ & $\begin{array}{l}6 \\
(17.1)\end{array}$ & \multirow[t]{2}{*}{2.825} & \multirow{2}{*}{ <. } & \multirow{2}{*}{$\begin{array}{l}\text { Reference } \\
3.96 \\
(1.62,11.25)\end{array}$} & $\begin{array}{l}53 \\
(438)\end{array}$ & & \multirow[t]{2}{*}{2.715} & \multirow{2}{*}{ <. } & Reference \\
\hline & $\begin{array}{l}61 \\
(55.0)\end{array}$ & $\begin{array}{l}29 \\
(82.9)\end{array}$ & & & & $\begin{array}{l}68 \\
(56.2)\end{array}$ & $\begin{array}{l}22 \\
(88.0)\end{array}$ & & & $\begin{array}{l}5.72(1.85, \\
25.06)\end{array}$ \\
\hline \multirow{2}{*}{$\begin{array}{l}\text { Gender } \\
\text { Male } \\
\text { Female }\end{array}$} & $\begin{array}{l}59 \\
(53.2)\end{array}$ & $\begin{array}{l}20 \\
(57.1)\end{array}$ & \multirow[t]{2}{*}{-0.413} & \multirow[t]{2}{*}{0.68} & \multirow{2}{*}{$\begin{array}{l}\text { Reference } \\
0.85 \\
(0.39,1.82)\end{array}$} & $\begin{array}{l}65 \\
(53.7)\end{array}$ & $\begin{array}{l}14 \\
(56.0)\end{array}$ & \multirow[t]{2}{*}{-0.208} & \multirow[t]{2}{*}{0.84} & Reference \\
\hline & $\begin{array}{l}52 \\
(46.8)\end{array}$ & $\begin{array}{l}15 \\
(42.9)\end{array}$ & & & & $\begin{array}{l}56 \\
(46.3)\end{array}$ & $\begin{array}{l}11 \\
(44.0)\end{array}$ & & & $\begin{array}{l}0.91 \\
(0.38,2.16)\end{array}$ \\
\hline \multirow{2}{*}{$\begin{array}{l}\text { Smoking } \\
\text { No } \\
\text { Yes }\end{array}$} & $\begin{array}{l}100 \\
(90.1)\end{array}$ & $\begin{array}{l}34 \\
(97.1)\end{array}$ & \multirow[t]{2}{*}{-1.241} & \multirow[t]{2}{*}{0.22} & \multirow{2}{*}{$\begin{array}{l}\text { Reference } \\
0.267 \\
(0.01,1.45)\end{array}$} & $\begin{array}{l}109 \\
(90.1)\end{array}$ & $\begin{array}{l}25 \\
(100.0)\end{array}$ & \multirow[t]{2}{*}{1.208} & \multirow[t]{2}{*}{0.23} & Reference \\
\hline & $\begin{array}{l}11 \\
(9.9)\end{array}$ & $1(2.9)$ & & & & $\begin{array}{l}12 \\
(9.9)\end{array}$ & $0(0.0)$ & & & $\begin{array}{l}0.17(0.01, \\
3.00)\end{array}$ \\
\hline BMI & $5(4.5)$ & $0(0)$ & -0.015 & 0.99 & Reference & $5(4.1)$ & $0(0)$ & -0.014 & 0.99 & Reference \\
\hline $\begin{array}{l}\text { Normal (18.5- } \\
22.9)\end{array}$ & $\begin{array}{l}19 \\
(17.1)\end{array}$ & $\begin{array}{l}7 \\
(20.0)\end{array}$ & & 0.35 & $\begin{array}{l}4.2(0.2 \\
86.3)\end{array}$ & $\begin{array}{l}20 \\
(16.5)\end{array}$ & $\begin{array}{l}6 \\
(24.0)\end{array}$ & & 0.42 & $\begin{array}{l}3.5(0.17 \\
72.00)\end{array}$ \\
\hline $\begin{array}{l}\text { Underweight (< } \\
\text { 18.5) }\end{array}$ & $\begin{array}{l}17 \\
(15.3)\end{array}$ & $\begin{array}{l}4 \\
(11.4)\end{array}$ & 0.94 & 0.35 & $\begin{array}{l}2.8(0.1 \\
61.2)\end{array}$ & $\begin{array}{l}18 \\
(14.9)\end{array}$ & $\begin{array}{l}3 \\
(12.0)\end{array}$ & 0.75 & 0.46 & $\begin{array}{l}2.1(0.09 \\
46.80)\end{array}$ \\
\hline $\begin{array}{l}\text { Overweight } \\
\text { (23.0-24.9) }\end{array}$ & $\begin{array}{l}41 \\
(36.9)\end{array}$ & $\begin{array}{l}15 \\
(42.9)\end{array}$ & 0.83 & 0.41 & $\begin{array}{l}4.1(0.2 \\
78.8)\end{array}$ & $\begin{array}{l}44 \\
(36.4)\end{array}$ & $\begin{array}{l}12 \\
(48.0)\end{array}$ & 0.231 & 0.82 & $\begin{array}{l}3.1(0.16 \\
60.00)\end{array}$ \\
\hline $\begin{array}{l}\text { Obese } 1 \text { (25.0- } \\
29.9)\end{array}$ & $\begin{array}{l}29 \\
(26.1)\end{array}$ & $\begin{array}{l}9 \\
(25.7)\end{array}$ & & & $\begin{array}{l}3.5(0.18 \\
70.2)\end{array}$ & $\begin{array}{l}34 \\
(28.1)\end{array}$ & $\begin{array}{l}4 \\
(16.0)\end{array}$ & & & $\begin{array}{l}1.4(0.07 \\
30.50)\end{array}$ \\
\hline Obese $2(>30.0)$ & & & & & & & & & & \\
\hline & $\begin{array}{l}86 \\
(77.5)\end{array}$ & $\begin{array}{l}23 \\
(65.7)\end{array}$ & 1.385 & 0.17 & & $\begin{array}{l}93 \\
(76.9)\end{array}$ & $\begin{array}{l}16 \\
(64.0)\end{array}$ & 1.332 & 0.18 & \\
\hline $\begin{array}{l}\text { No } \\
\text { Yes }\end{array}$ & $\begin{array}{l}25 \\
(22.5)\end{array}$ & $\begin{array}{l}12 \\
(34.3)\end{array}$ & & & $\begin{array}{l}1.79 \\
(0.77,4.08)\end{array}$ & $\begin{array}{l}28 \\
(23.1)\end{array}$ & $\begin{array}{l}9 \\
(36.0)\end{array}$ & & & $\begin{array}{l}1.87 \\
(0.72,4.63)\end{array}$ \\
\hline Gout & $\begin{array}{l}100 \\
(90.1)\end{array}$ & $\begin{array}{l}33 \\
(94.3)\end{array}$ & -0.75 & 0.45 & Reference & $\begin{array}{l}110 \\
(90.9)\end{array}$ & $\begin{array}{l}23 \\
(92.0)\end{array}$ & -0.174 & 0.86 & Reference \\
\hline & $\begin{array}{l}11 \\
(9.9)\end{array}$ & $2(5.7)$ & & & & $\begin{array}{l}11 \\
(9.1)\end{array}$ & $2(0.8)$ & & & \\
\hline $\begin{array}{l}\text { No } \\
\text { Yes }\end{array}$ & & & & & $\begin{array}{l}0.55 \\
(0.08,2.19)\end{array}$ & & & & & $\begin{array}{l}0.87 \\
(0.13,3.53)\end{array}$ \\
\hline Hyperlipidaemia & $\begin{array}{l}52 \\
(46.8)\end{array}$ & $\begin{array}{l}13 \\
(37.1)\end{array}$ & 1.004 & 0.32 & Reference & $\begin{array}{l}54 \\
(44.6)\end{array}$ & $\begin{array}{l}11 \\
(44.0)\end{array}$ & 0.058 & 0.95 & Reference \\
\hline Yes & $\begin{array}{l}59 \\
(53.2)\end{array}$ & $\begin{array}{l}22 \\
(62.9)\end{array}$ & & & $\begin{array}{l}1.49 \\
(0.69,3.32)\end{array}$ & $\begin{array}{l}67 \\
(55.4)\end{array}$ & $\begin{array}{l}14 \\
(56.0)\end{array}$ & & & $\begin{array}{l}1.02(0.43, \\
2.49)\end{array}$ \\
\hline Stroke & $\begin{array}{l}105 \\
(94.6)\end{array}$ & $\begin{array}{l}34 \\
(97.1)\end{array}$ & -0.605 & 0.55 & Reference & $\begin{array}{l}114 \\
(94.2)\end{array}$ & $\begin{array}{l}25 \\
(100)\end{array}$ & 0.817 & 0.41 & Reference \\
\hline $\begin{array}{l}\text { No } \\
\text { Yes }\end{array}$ & $6(5.4)$ & $1(2.9)$ & & & $\begin{array}{l}0.51 \\
(0.03,3.16)\end{array}$ & $7(5.8)$ & $0(100)$ & & & $\begin{array}{l}0.30(0.17, \\
5.40)\end{array}$ \\
\hline
\end{tabular}

*Factor significant at $\mathrm{P}<0.15$ to be included in multiple logistic regression. 


\begin{tabular}{|c|c|c|c|c|c|c|c|c|c|c|}
\hline & \multicolumn{2}{|l|}{ SD } & \multirow{2}{*}{$\begin{array}{l}\text { Test } \\
\text { statistic }\end{array}$} & \multirow{2}{*}{$\begin{array}{l}\text { p- } \\
\text { value }\end{array}$} & \multirow{2}{*}{$\begin{array}{l}\text { Crude Odds } \\
\text { ratio (95\% } \\
\text { Cl) }\end{array}$} & \multicolumn{2}{|l|}{$\% \mathrm{CV}$} & \multirow{2}{*}{$\begin{array}{l}\text { Test } \\
\text { statistic }\end{array}$} & \multirow{2}{*}{$\begin{array}{l}\text { p- } \\
\text { value }\end{array}$} & \multirow{2}{*}{$\begin{array}{l}\text { Crude } \\
\text { Odds ratio } \\
(95 \% \mathrm{Cl})\end{array}$} \\
\hline & Target & High & & & & Target & High & & & \\
\hline Hypertension & $4(3.6)$ & $0(0)$ & 0.725 & 0.47 & Reference & $4(3.3)$ & $0(0)$ & 0.444 & 0.66 & Reference \\
\hline \multirow{2}{*}{$\begin{array}{l}\text { No } \\
\text { Yes }\end{array}$} & $\begin{array}{l}107 \\
(96.4)\end{array}$ & $\begin{array}{l}35 \\
(100)\end{array}$ & & & $\begin{array}{l}3.00(0.16 \\
56.6)\end{array}$ & $\begin{array}{l}117 \\
(96.7)\end{array}$ & $\begin{array}{l}25 \\
(100)\end{array}$ & & & $\begin{array}{l}2.0(0.10 \\
37.40)\end{array}$ \\
\hline & \multicolumn{5}{|c|}{ Mean (SD) } & \multicolumn{5}{|c|}{ Mean (SD) } \\
\hline Age & $\begin{array}{l}53.1 \\
(11.8)\end{array}$ & $\begin{array}{l}58.4 \\
(11.8)\end{array}$ & 2.236 & $0.03^{*}$ & $\begin{array}{l}1.04 \\
(1.00,1.08)\end{array}$ & $\begin{array}{l}53.1 \\
(11.8)\end{array}$ & $\begin{array}{l}58.4 \\
(11.8)\end{array}$ & 3.06 & <. $01 *$ & $\begin{array}{l}1.07 \\
(1.03,1.12)\end{array}$ \\
\hline Duration HD & $\begin{array}{l}4.1 \\
(3.2)\end{array}$ & $\begin{array}{l}4.0 \\
(3.2)\end{array}$ & -0.0237 & 0.81 & $\begin{array}{l}0.99 \\
(0.87,1.11)\end{array}$ & $\begin{array}{l}4.1 \\
(3.2)\end{array}$ & $\begin{array}{l}4.0 \\
(3.2)\end{array}$ & -0.215 & 0.83 & $\begin{array}{l}0.99 \\
(0.85,1.12)\end{array}$ \\
\hline BMI $\left(\mathrm{kg} / \mathrm{m}^{2}\right)$ & $\begin{array}{l}26.7 \\
(5.4)\end{array}$ & $\begin{array}{l}27.1 \\
(5.2)\end{array}$ & 0.365 & 0.72 & $\begin{array}{l}1.01 \\
(0.94,1.09)\end{array}$ & $\begin{array}{l}26.7 \\
(5.4)\end{array}$ & $\begin{array}{l}27.1 \\
(5.2)\end{array}$ & -0.727 & 0.47 & $\begin{array}{l}0.97(0.89, \\
1.05)\end{array}$ \\
\hline HbA1c & $\begin{array}{l}6.4 \\
(1.6)\end{array}$ & $\begin{array}{l}7.4 \\
(1.6)\end{array}$ & 3.030 & <. $01 *$ & $\begin{array}{l}1.42 \\
(1.13,1.79)\end{array}$ & $\begin{array}{l}6.4 \\
(1.6)\end{array}$ & $\begin{array}{l}7.4 \\
(1.6)\end{array}$ & 1.664 & $0.10 *$ & $\begin{array}{l}1.23 \\
(0.96,1.57)\end{array}$ \\
\hline HSCRP & $\begin{array}{l}7.3 \\
(7.0)\end{array}$ & $\begin{array}{l}9.0 \\
(9.8)\end{array}$ & 1.11 & 0.27 & $\begin{array}{l}1.02(0.98, \\
1.07)\end{array}$ & $\begin{array}{l}7.3 \\
(7.0)\end{array}$ & $\begin{array}{l}9.0 \\
(4.8)\end{array}$ & 0.63 & 0.53 & $\begin{array}{l}1.02(0.96, \\
1.07)\end{array}$ \\
\hline Ferritin & $\begin{array}{l}632.9 \\
(424.8)\end{array}$ & $\begin{array}{l}498.6 \\
(372.1)\end{array}$ & -1.6 & $0.11 *$ & $\begin{array}{l}0.99 \\
(0.99,1.00)\end{array}$ & $\begin{array}{l}632.9 \\
(424.8)\end{array}$ & $\begin{array}{l}498.6 \\
(372.1)\end{array}$ & -1.765 & $0.08 *$ & $\begin{array}{l}0.99(0.99, \\
1.00)\end{array}$ \\
\hline Albumin & $\begin{array}{l}38.9 \\
(3.9)\end{array}$ & $\begin{array}{l}38.9 \\
(3.4)\end{array}$ & 0.132 & 0.90 & $\begin{array}{l}1.01 \\
(0.91,1.12)\end{array}$ & $\begin{array}{l}38.9 \\
(3.9)\end{array}$ & $\begin{array}{l}38.9 \\
(3.4)\end{array}$ & -1.203 & 0.23 & $\begin{array}{l}0.94 \\
(0.84,1.05)\end{array}$ \\
\hline Clearance & $\begin{array}{l}69.2 \\
(8.8)\end{array}$ & $\begin{array}{l}70.1 \\
(10.4)\end{array}$ & 0.5 & 0.62 & $\begin{array}{l}1.01 \\
(0.97,1.06)\end{array}$ & $\begin{array}{l}69.2 \\
(8.8)\end{array}$ & $\begin{array}{l}70.1 \\
(10.5)\end{array}$ & 0.64 & 0.52 & $\begin{array}{l}1.01(0.97, \\
1.07)\end{array}$ \\
\hline LDL & $\begin{array}{l}3.1 \\
(1.1)\end{array}$ & $\begin{array}{l}2.7 \\
(1.2)\end{array}$ & -1.488 & $0.14^{*}$ & $\begin{array}{l}0.76 \\
(0.53,1.08)\end{array}$ & $\begin{array}{l}3.1 \\
(1.1)\end{array}$ & $\begin{array}{l}2.7 \\
(1.2)\end{array}$ & -1.525 & $0.13^{*}$ & $\begin{array}{l}0.72(0.47, \\
1.08)\end{array}$ \\
\hline HDL & $\begin{array}{l}1.01 \\
(0.26)\end{array}$ & $\begin{array}{l}1 \\
(0.24)\end{array}$ & -0.191 & 0.85 & $\begin{array}{l}0.86 \\
(0.18,3.83)\end{array}$ & $\begin{array}{l}1.01 \\
(0.3)\end{array}$ & $1(0.2)$ & -0.241 & 0.81 & $\begin{array}{l}0.81(0.13, \\
4.35)\end{array}$ \\
\hline HB & $\begin{array}{l}10.4 \\
(1.6)\end{array}$ & $\begin{array}{l}10.6 \\
(1.9)\end{array}$ & 0.76 & 0.45 & $\begin{array}{l}1.09 \\
(0.87,1.36)\end{array}$ & $\begin{array}{l}10.4 \\
(1.6)\end{array}$ & $\begin{array}{l}10.6 \\
(1.9)\end{array}$ & -0.014 & 0.99 & $\begin{array}{l}0.99(0.77, \\
1.28)\end{array}$ \\
\hline TG & $\begin{array}{l}2.1 \\
(1.5)\end{array}$ & $\begin{array}{l}2.6 \\
(2.0)\end{array}$ & 1.431 & $0.15^{*}$ & $\begin{array}{l}1.17 \\
(0.94,1.45)\end{array}$ & $\begin{array}{l}2.1 \\
(1.5)\end{array}$ & $\begin{array}{l}2.6 \\
(2.0)\end{array}$ & 0.883 & 0.38 & $\begin{array}{l}1.11 \\
(0.86,1.40)\end{array}$ \\
\hline $\begin{array}{l}\text { Transferrin } \\
\text { saturation }\end{array}$ & $\begin{array}{l}24.7 \\
(10.4)\end{array}$ & $\begin{array}{l}23.3 \\
(9.9)\end{array}$ & -0.71 & 0.48 & $\begin{array}{l}0.99 \\
(0.95,1.02)\end{array}$ & $\begin{array}{l}24.7 \\
(10.4)\end{array}$ & $\begin{array}{l}23.3 \\
(9.9)\end{array}$ & -1.217 & 0.22 & $\begin{array}{l}0.97(0.92, \\
1.02)\end{array}$ \\
\hline Calcium & $\begin{array}{l}2.2 \\
(0.2)\end{array}$ & $\begin{array}{l}2.2 \\
(0.21)\end{array}$ & -0.186 & 0.85 & $\begin{array}{l}0.85 \\
(0.16,4.94)\end{array}$ & $\begin{array}{l}2.2 \\
(0.2)\end{array}$ & $\begin{array}{l}2.2 \\
(0.2)\end{array}$ & -0.441 & 0.66 & $\begin{array}{l}1.6(0.23 \\
12.6)\end{array}$ \\
\hline Phosphate & $\begin{array}{l}2.0 \\
(0.6)\end{array}$ & $\begin{array}{l}2.0 \\
(0.7)\end{array}$ & -0.193 & 0.85 & $\begin{array}{l}0.94 \\
(0.51,1.70)\end{array}$ & $\begin{array}{l}1.9 \\
(0.6)\end{array}$ & $\begin{array}{l}1.9 \\
(0.7)\end{array}$ & -1.42 & 0.16 & $\begin{array}{l}0.59 \\
1.8)\end{array}$ \\
\hline ALP & $\begin{array}{l}151.8 \\
(109.0)\end{array}$ & $\begin{array}{l}176.1 \\
(166.4)\end{array}$ & 0.99 & 0.32 & $\begin{array}{l}1.00(0.99 \\
1.00)\end{array}$ & $\begin{array}{l}151.8 \\
(109.0)\end{array}$ & $\begin{array}{l}176.1 \\
(166.4)\end{array}$ & 1.847 & 0.17 & $\begin{array}{l}1.00 \\
(1.00,1.01)\end{array}$ \\
\hline iPTH & $\begin{array}{l}87.5 \\
(82.3)\end{array}$ & $\begin{array}{l}80.1 \\
(79.0)\end{array}$ & -0.44 & 0.66 & $\begin{array}{l}0.99 \\
(0.99,1.00)\end{array}$ & $\begin{array}{l}87.5 \\
(83.0)\end{array}$ & $\begin{array}{l}80.0 \\
(79.0)\end{array}$ & 0.297 & 0.77 & $\begin{array}{l}1.00 \\
(0.99,1.01)\end{array}$ \\
\hline
\end{tabular}


Table 4

shows simple logistic regression analysis of socio-demographic, clinical comorbidities and blood parameters among patients in cohort $(n=150)$ with poor GV during NHD.

\begin{tabular}{|c|c|c|c|c|c|c|c|c|c|c|}
\hline & \multicolumn{2}{|l|}{ SD } & \multirow{2}{*}{$\begin{array}{l}\text { Test } \\
\text { statistic }\end{array}$} & \multirow{2}{*}{$\begin{array}{l}\mathrm{P} \\
\text { Value }\end{array}$} & \multirow{2}{*}{$\begin{array}{l}\text { Crude } \\
\text { Odds ratio } \\
(95 \% \mathrm{Cl})\end{array}$} & \multicolumn{2}{|l|}{$\% \mathrm{CV}$} & \multirow{2}{*}{$\begin{array}{l}\text { Test } \\
\text { statistic }\end{array}$} & \multirow{2}{*}{$\begin{array}{l}\mathrm{P} \\
\text { Value }\end{array}$} & \multirow{2}{*}{$\begin{array}{l}\text { Crude Odds } \\
\text { ratio ( } 95 \% \\
\mathrm{Cl})\end{array}$} \\
\hline & Target & High & & & & Target & High & & & \\
\hline \multirow{3}{*}{$\begin{array}{l}\text { Diabetes } \\
\text { No } \\
\text { Yes }\end{array}$} & 57 & $0(0)$ & \multirow[t]{3}{*}{1.866} & \multirow[t]{3}{*}{$0.06 *$} & Reference & \multirow{2}{*}{$\begin{array}{l}57 \\
(41.0)\end{array}$} & $0(0)$ & \multirow[t]{3}{*}{1.596} & \multirow[t]{3}{*}{$0.11 *$} & Reference \\
\hline & & 10 & & & \multirow{2}{*}{$\begin{array}{l}15.2(0.88 \\
264.5)\end{array}$} & & \multirow{2}{*}{$7(100)$} & & & 10.5 (0.59, \\
\hline & $\begin{array}{l}79 \\
(58.1)\end{array}$ & $(100)$ & & & & $\begin{array}{l}82 \\
(59.0)\end{array}$ & & & & 186.70) \\
\hline \multirow{3}{*}{$\begin{array}{l}\text { Gender } \\
\text { Male } \\
\text { Female }\end{array}$} & $\begin{array}{l}76 \\
(55.9)\end{array}$ & $\begin{array}{l}5 \\
(50.0)\end{array}$ & \multirow[t]{3}{*}{0.361} & \multirow[t]{3}{*}{0.72} & \multirow{3}{*}{$\begin{array}{l}\text { Reference } \\
1.27(0.34 \\
4.75)\end{array}$} & 77 & $\begin{array}{l}4 \\
(571)\end{array}$ & \multirow[t]{3}{*}{-0.91} & \multirow[t]{3}{*}{0.93} & Reference \\
\hline & & & & & & & & & & $0.93(0.18$ \\
\hline & $(44.1)$ & $\begin{array}{l}5 \\
(50.0)\end{array}$ & & & & $(44.6)$ & $(42.9)$ & & & \\
\hline \multirow{3}{*}{$\begin{array}{l}\text { Smoking } \\
\text { No } \\
\text { Yes }\end{array}$} & $\begin{array}{l}122 \\
(89.7)\end{array}$ & $\begin{array}{l}10 \\
(100)\end{array}$ & \multirow[t]{3}{*}{0.516} & \multirow[t]{3}{*}{0.61} & \multirow{3}{*}{$\begin{array}{l}\text { Reference } \\
0.50(0.02, \\
8.50)\end{array}$} & \multirow{3}{*}{$\begin{array}{l}125 \\
(89.9) \\
14 \\
(10.1)\end{array}$} & $7(100)$ & \multirow[t]{3}{*}{0.37} & \multirow[t]{3}{*}{0.71} & Reference \\
\hline & & & & & & & $0(0)$ & & & $0.58(0.03$ \\
\hline & $\begin{array}{l}12 \\
(10.3)\end{array}$ & $0(0)$ & & & & & & & & 10.60) \\
\hline BMI & $5(3.7)$ & $0(0)$ & -.009 & 0.992 & Reference & $5(3.6)$ & $0(0)$ & -0.004 & 0.99 & Reference \\
\hline $\begin{array}{l}\text { Normal (18.5- } \\
22.9)\end{array}$ & $\begin{array}{l}24 \\
(17.7)\end{array}$ & $\begin{array}{l}2 \\
(20.0)\end{array}$ & & 0.94 & $\begin{array}{l}1.1(0.05 \\
26.80)\end{array}$ & $\begin{array}{l}26 \\
(18.7)\end{array}$ & $0(0)$ & 0.77 & 0.44 & $0.2(0.01$ \\
\hline $\begin{array}{l}\text { Underweight (< } \\
18.5 \text { ) }\end{array}$ & $\begin{array}{l}21 \\
(15.4)\end{array}$ & $\begin{array}{l}1 \\
(10.0)\end{array}$ & 0.22 & 0.83 & $\begin{array}{l}0.77(0.03 \\
21.50)\end{array}$ & $\begin{array}{l}21 \\
(15.1)\end{array}$ & $\begin{array}{l}1 \\
(14.3)\end{array}$ & $\begin{array}{l}0.16 \\
0.44\end{array}$ & 0.66 & $\begin{array}{l}0.7(0.02 \\
21.5)\end{array}$ \\
\hline $\begin{array}{l}\text { Overweight } \\
(23.0-24.9)\end{array}$ & $\begin{array}{l}54 \\
(39.7)\end{array}$ & $\begin{array}{l}3 \\
(30.0)\end{array}$ & 0.27 & 0.78 & $\begin{array}{l}0.71(0.03 \\
15.50)\end{array}$ & $\begin{array}{l}55 \\
(39.6)\end{array}$ & $(28.6)$ & 0.27 & 0.79 & $\begin{array}{l}0.5(0.02 \\
11.7)\end{array}$ \\
\hline $\begin{array}{l}\text { Obese } 1 \text { ( } 25.0- \\
29.9)\end{array}$ & $\begin{array}{l}32 \\
(23.5)\end{array}$ & $\begin{array}{l}4 \\
(40.0)\end{array}$ & & & $\begin{array}{l}1.5(0.07 \\
32.40)\end{array}$ & $\begin{array}{l}32 \\
(23.0)\end{array}$ & $\begin{array}{l}4 \\
(57.1)\end{array}$ & & & $\begin{array}{l}1.5(0.07 \\
32.4)\end{array}$ \\
\hline Obese $2(>30.0)$ & & & & & & & & & & \\
\hline IHD & $\begin{array}{l}99 \\
(72.8)\end{array}$ & $\begin{array}{l}10 \\
(100.0)\end{array}$ & 1.417 & 0.16 & Reference & 102 & $7(100)$ & 1.156 & 0.25 & Reference \\
\hline Yes & $\begin{array}{l}37 \\
(27.2)\end{array}$ & $0(0)$ & & & $\begin{array}{l}0.13(0.01 \\
2.20)\end{array}$ & $\begin{array}{l}37 \\
(26.6)\end{array}$ & $0(0)$ & & & $\begin{array}{l}0.18 \\
(0.01,3.30)\end{array}$ \\
\hline Gout & $\begin{array}{l}123 \\
(90.4)\end{array}$ & $\begin{array}{l}10 \\
(100.0)\end{array}$ & 0.563 & 0.57 & Reference & $\begin{array}{l}126 \\
(90.6)\end{array}$ & $7(100)$ & 0.316 & 0.75 & Reference \\
\hline $\begin{array}{l}\text { No } \\
\text { Yes }\end{array}$ & $\begin{array}{l}13 \\
(9.6)\end{array}$ & $0(0)$ & & & $\begin{array}{l}0.44(0.02, \\
7.80)\end{array}$ & $\begin{array}{l}13 \\
(9.4)\end{array}$ & $0(0)$ & & & $\begin{array}{l}0.62(0.03 \\
11.60)\end{array}$ \\
\hline Hyperlipidaemia & $\begin{array}{l}61 \\
(44.9)\end{array}$ & $2(20)$ & 1.458 & $0.15 *$ & Reference & $\begin{array}{l}62 \\
(44.6)\end{array}$ & $\begin{array}{l}1 \\
(14.3)\end{array}$ & 1.441 & $0.15^{\star}$ & Reference \\
\hline $\begin{array}{l}\text { No } \\
\text { Yes }\end{array}$ & $\begin{array}{l}75 \\
(55.1)\end{array}$ & $8(80)$ & & & $\begin{array}{l}3.25(0.78 \\
22.10)\end{array}$ & $\begin{array}{l}77 \\
(55.4)\end{array}$ & $\begin{array}{l}6 \\
(85.7)\end{array}$ & & & $\begin{array}{l}4.8(0.80 \\
92.50)\end{array}$ \\
\hline Stroke & $\begin{array}{l}129 \\
(94.9)\end{array}$ & $\begin{array}{l}10 \\
(100)\end{array}$ & 0.131 & 0.90 & Reference & 132 & $7(100)$ & 0.202 & 0.840 & Reference \\
\hline $\begin{array}{l}\text { No } \\
\text { Yes }\end{array}$ & $7(5.1)$ & $0(0)$ & & & $\begin{array}{l}0.82(0.04 \\
15.40)\end{array}$ & $6(5.0)$ & $0(0)$ & & & $\begin{array}{l}1.36(0.07 \\
26.50)\end{array}$ \\
\hline Hypertension & $4(2.9)$ & $0(0)$ & 0.222 & 0.82 & Reference & $4(2.9)$ & $0(0)$ & 0.453 & 0.650 & Reference \\
\hline No & $\begin{array}{l}132 \\
(97.1)\end{array}$ & $\begin{array}{l}10 \\
(100)\end{array}$ & & & $\begin{array}{l}0.71(0.04 \\
14.20)\end{array}$ & $\begin{array}{l}135 \\
(97.1)\end{array}$ & $7(100)$ & & & $\begin{array}{l}0.50 \\
(0.02,10.10)\end{array}$ \\
\hline & Mean ( & & & & & Mean ( & & & & \\
\hline
\end{tabular}




\begin{tabular}{|c|c|c|c|c|c|c|c|c|c|c|}
\hline & \multicolumn{2}{|l|}{ SD } & \multirow{2}{*}{$\begin{array}{l}\text { Test } \\
\text { statistic }\end{array}$} & \multirow{2}{*}{$\begin{array}{l}\mathrm{P} \\
\text { Value }\end{array}$} & \multirow{2}{*}{$\begin{array}{l}\text { Crude } \\
\text { Odds ratio } \\
(95 \% \mathrm{Cl})\end{array}$} & \multicolumn{2}{|l|}{$\% \mathrm{CV}$} & \multirow{2}{*}{$\begin{array}{l}\text { Test } \\
\text { statistic }\end{array}$} & \multirow{2}{*}{$\begin{array}{l}\mathrm{P} \\
\text { Value }\end{array}$} & \multirow{2}{*}{$\begin{array}{l}\text { Crude Odds } \\
\text { ratio ( } 95 \% \\
\mathrm{Cl})\end{array}$} \\
\hline & Target & High & & & & Target & High & & & \\
\hline Age & $\begin{array}{l}53.1 \\
(11.8)\end{array}$ & $\begin{array}{l}58.4 \\
(11.8)\end{array}$ & 2.135 & $0.03^{*}$ & $\begin{array}{l}1.07(1.00 \\
1.40)\end{array}$ & $\begin{array}{l}53.1 \\
(11.8)\end{array}$ & $\begin{array}{l}58.4 \\
(11.8)\end{array}$ & 1.846 & $0.06 *$ & $\begin{array}{l}1.07 \\
(0.99,1.15)\end{array}$ \\
\hline Duration HD & $\begin{array}{l}4.1 \\
(3.2)\end{array}$ & $\begin{array}{l}4.0 \\
(3.2)\end{array}$ & 0.428 & 0.67 & $\begin{array}{l}1.04(0.84 \\
1.26)\end{array}$ & $\begin{array}{l}4.1 \\
(3.2)\end{array}$ & $\begin{array}{l}4.0 \\
(3.2)\end{array}$ & 0.678 & 0.50 & $\begin{array}{l}1.08(0.84 \\
1.34)\end{array}$ \\
\hline BMI $\left(\mathrm{kg} / \mathrm{m}^{2}\right)$ & $\begin{array}{l}26.7 \\
(5.4)\end{array}$ & $\begin{array}{l}27.1 \\
(5.2)\end{array}$ & 0.50 & 0.62 & $\begin{array}{l}1.03(0.91 \\
1.16)\end{array}$ & $\begin{array}{l}26.7 \\
(5.4)\end{array}$ & $\begin{array}{l}27.1 \\
(5.2)\end{array}$ & 1.56 & 0.17 & $\begin{array}{l}1.11(0.97 \\
1.28)\end{array}$ \\
\hline HbA1c & $\begin{array}{l}6.4 \\
(1.6)\end{array}$ & $\begin{array}{l}7.4 \\
(1.6)\end{array}$ & 2.32 & $0.02 *$ & $\begin{array}{l}1.49(1.06, \\
2.10)\end{array}$ & $\begin{array}{l}6.4 \\
(1.6)\end{array}$ & $\begin{array}{l}7.4 \\
(1.6)\end{array}$ & 2.70 & j.01* & $\begin{array}{l}1.73(1.17 \\
2.64)\end{array}$ \\
\hline HSCRP & $\begin{array}{l}7.3 \\
(7.0)\end{array}$ & $\begin{array}{l}9.0 \\
(9.8)\end{array}$ & -0.689 & 0.49 & $\begin{array}{l}0.96(0.85, \\
1.05)\end{array}$ & $\begin{array}{l}7.3 \\
(7.0)\end{array}$ & $\begin{array}{l}9.0 \\
(9.8)\end{array}$ & -0.414 & 0.68 & $\begin{array}{l}0.98(0.84, \\
1.07)\end{array}$ \\
\hline Ferritin & $\begin{array}{l}632.9 \\
(424.8)\end{array}$ & $\begin{array}{l}498.6 \\
(372.1)\end{array}$ & -1.165 & 0.25 & $\begin{array}{l}0.99(0.99 \\
1.00)\end{array}$ & $\begin{array}{l}632.9 \\
(424.8)\end{array}$ & $\begin{array}{l}498.6 \\
(372.1)\end{array}$ & -1.740 & $0.08 *$ & $\begin{array}{l}0.99 \\
(0.99,0.99)\end{array}$ \\
\hline Albumin & $\begin{array}{l}38.9 \\
(3.9)\end{array}$ & $\begin{array}{l}38.9 \\
(3.4)\end{array}$ & 1.776 & $0.08 *$ & $\begin{array}{l}1.2(0.99 \\
1.50)\end{array}$ & $\begin{array}{l}38.9 \\
(3.9)\end{array}$ & $\begin{array}{l}38.9 \\
(3.3)\end{array}$ & 1.478 & $0.14^{*}$ & $\begin{array}{l}1.12(0.96 \\
1.54)\end{array}$ \\
\hline Clearance & $\begin{array}{l}69.2 \\
(8.8)\end{array}$ & $\begin{array}{l}70.1 \\
(10.4)\end{array}$ & 2.003 & 0.30 & $\begin{array}{l}1.08(1.00 \\
1.18)\end{array}$ & $\begin{array}{l}69.2 \\
(8.8)\end{array}$ & $\begin{array}{l}70.1 \\
(10.4)\end{array}$ & 0.606 & 0.55 & $\begin{array}{l}1.03(0.95 \\
1.12)\end{array}$ \\
\hline LDL & $\begin{array}{l}3.1 \\
(1.1)\end{array}$ & $\begin{array}{l}2.7 \\
(1.2)\end{array}$ & 0.230 & 0.82 & $\begin{array}{l}1.07(0.58, \\
1.88)\end{array}$ & $\begin{array}{l}3.08 \\
(1.1)\end{array}$ & $\begin{array}{l}2.7 \\
(1.2)\end{array}$ & 0.525 & 0.60 & $\begin{array}{l}1.19(0.61, \\
2.23)\end{array}$ \\
\hline HDL & $\begin{array}{l}1.0 \\
(0.3)\end{array}$ & $1(0.2)$ & 0.055 & 0.96 & $\begin{array}{l}1.07(0.07 \\
11.8)\end{array}$ & $\begin{array}{l}1.0 \\
(0.3)\end{array}$ & $1(0.2)$ & 0.359 & 0.72 & $\begin{array}{l}1.7(0.07 \\
26.2)\end{array}$ \\
\hline HB & $\begin{array}{l}10.4 \\
(1.6)\end{array}$ & $\begin{array}{l}10.6 \\
(2.0)\end{array}$ & -0.468 & 0.64 & $\begin{array}{l}0.91 \\
1.32)\end{array}$ & $\begin{array}{l}10.4 \\
(1.6)\end{array}$ & $\begin{array}{l}10.6 \\
(1.9)\end{array}$ & 0.659 & 0.51 & $\begin{array}{l}1.15(0.7 \\
1.74)\end{array}$ \\
\hline TG & $\begin{array}{l}2.1 \\
(1.5)\end{array}$ & $\begin{array}{l}2.6 \\
(2.0)\end{array}$ & 1.025 & 0.31 & $\begin{array}{l}1.17(0.82, \\
1.55)\end{array}$ & $\begin{array}{l}2.1 \\
(1.5)\end{array}$ & $\begin{array}{l}2.6 \\
(2.0)\end{array}$ & -0.168 & 0.87 & $\begin{array}{l}0.96(0.49 \\
1.42)\end{array}$ \\
\hline $\begin{array}{l}\text { Transferrin } \\
\text { saturation }\end{array}$ & $\begin{array}{l}24.7 \\
(10.4)\end{array}$ & $\begin{array}{l}23.3 \\
(9.9)\end{array}$ & 0.655 & 0.51 & $\begin{array}{l}1.02(0.96 \\
1.08)\end{array}$ & $\begin{array}{l}24.7 \\
(10.4)\end{array}$ & $\begin{array}{l}23.3 \\
(9.9)\end{array}$ & -0.09 & 0.93 & $\begin{array}{l}0.99 \\
(0.92,1.07)\end{array}$ \\
\hline Calcium & $\begin{array}{l}2.2 \\
(0.2)\end{array}$ & $\begin{array}{l}2.2 \\
(0.2)\end{array}$ & -0.522 & 0.60 & $\begin{array}{l}0.48(0.03 \\
9.20)\end{array}$ & $\begin{array}{l}2.2 \\
(0.2)\end{array}$ & $\begin{array}{l}2.2 \\
(0.2)\end{array}$ & -0.843 & 0.40 & $\begin{array}{l}0.26(0.01 \\
7.7)\end{array}$ \\
\hline Phosphate & $\begin{array}{l}2.0 \\
(0.6)\end{array}$ & $\begin{array}{l}2.0 \\
(0.7)\end{array}$ & -1.992 & 0.17 & $\begin{array}{l}0.25(0.06 \\
0.86)\end{array}$ & $\begin{array}{l}2.0 \\
(0.6)\end{array}$ & $\begin{array}{l}2.0 \\
(0.7)\end{array}$ & -1.558 & 0.16 & $\begin{array}{l}0.29 \\
1.17)\end{array}$ \\
\hline ALP & $\begin{array}{l}151.8 \\
(109.0)\end{array}$ & $\begin{array}{l}176.1 \\
(166.0)\end{array}$ & -0.68 & 0.50 & $\begin{array}{l}0.99 \\
1.00)\end{array}$ & $\begin{array}{l}151.8 \\
(109.0)\end{array}$ & $\begin{array}{l}176.1 \\
(166.4)\end{array}$ & -0.451 & 0.65 & $\begin{array}{l}0.99(0.99 \\
1.00)\end{array}$ \\
\hline iPTH & $\begin{array}{l}87.5 \\
(83.0)\end{array}$ & $\begin{array}{l}80.1 \\
(79.0)\end{array}$ & -0.305 & 0.76 & $\begin{array}{l}0.99 \\
(0.99,1.01)\end{array}$ & $\begin{array}{l}87.5 \\
(83.0)\end{array}$ & $\begin{array}{l}80.1 \\
(79.0)\end{array}$ & -0.536 & 0.60 & $\begin{array}{l}0.99(0.98 \\
1.01)\end{array}$ \\
\hline
\end{tabular}


Table 5

shows multiple logistic regression analysis of significant factors associated with poor SD GV among ESKD patients during haemodialysis (HD) day. OR, odd ratio;

$R^{2}=0.22$ (Nagelkerke) $* p<0.05$.

\begin{tabular}{|llllll|}
\hline SD Variables & $\boldsymbol{\beta}$ & SE & Wald & Adjusted OR $(95 \% \mathrm{Cl})$ & P-Value \\
\hline Diabetes & 0.65 & 0.65 & 0.99 & $1.9(0.5,7.4)$ & 0.32 \\
\hline Age & 0.027 & 0.02 & 1.27 & $1.0(0.9,1.1)$ & 0.20 \\
\hline HBA1C & 0.26 & 0.16 & 1.60 & $1.3(0.9,1.8)$ & 0.11 \\
\hline Ferritin & -0.007 & 0.0006 & -1.06 & $0.9(0.9,1.00)$ & 0.29 \\
\hline LDL & -0.479 & $\mathbf{0 . 2 3 4}$ & -2.05 & $\mathbf{0 . 6}(0.4,0.9)$ & $\mathbf{0 . 0 4}$ \\
\hline TG & 0.248 & 0.157 & 1.58 & $1.3(0.9,1.7)$ & 0.11 \\
\hline \%CV Variables & $\boldsymbol{\beta}$ & $\mathrm{SE}$ & Wald & Adjusted OR $(95 \% \mathrm{Cl})$ & P-Value \\
\hline Diabetes & 1.13 & 0.76 & 1.48 & $3.1(0.7,16.2)$ & 0.14 \\
\hline Age & $\mathbf{0 . 0 5}$ & $\mathbf{0 . 0 2}$ & $\mathbf{2 . 0}$ & $\mathbf{1 . 0}(1.0,1.1)$ & $\mathbf{0 . 0 5}$ \\
\hline HBA1C & 0.03 & 0.17 & 0.19 & $1.0(0.7,1.4)$ & 0.85 \\
\hline Ferritin & -0.001 & 0.0008 & -1.54 & $0.9(0.9,1.0)$ & 0.12 \\
\hline LDL & -0.33 & 0.24 & -1.35 & $0.7(0.4,1.1)$ & 0.18 \\
\hline
\end{tabular}

Table 6

shows multiple logistic regression analysis of significant factors associated with poor \%CV GV among ESKD patients during non- haemodialysis (NHD) day. OR, odds ratio; $\mathrm{R}^{2}=0.31$ (Nagelkerke) ${ }^{*} p<0.05$.

\begin{tabular}{|llllll|}
\hline SD & $\beta$ & SE & Wald & Adjusted OR $(95 \%$ Cl $)$ & P-Value \\
\hline Variable & & & & & \\
\hline Diabetes & 18.23 & 2128.0 & 0.01 & $83.3(0.001$, Inf $)$ & 0.99 \\
\hline Age & 0.54 & 0.97 & 0.56 & $1.7(0.3,1.5)$ & 0.58 \\
\hline HBA1C & 0.03 & 0.04 & 0.75 & $1.0(0.9,1.1)$ & 0.46 \\
\hline Ferritin & 0.22 & 0.25 & 0.88 & $1.2(0.8,2.1)$ & 0.38 \\
\hline Albumin & $\mathbf{0 . 2 6}$ & $\mathbf{0 . 1 5}$ & $\mathbf{1 . 7 6}$ & $1.3(1.0,1.8)$ & $0.08 *$ \\
\hline \%CV & $\boldsymbol{\beta}$ & $\mathrm{SE}$ & Wald & Adjusted OR $(95 \% \mathrm{Cl})$ & P-Value \\
Variable & & & & & 0.99 \\
\hline Diabetes & 16.87 & 2224.6 & 0.008 & $21.1(0.03, \mathrm{Inf})$ & 0.44 \\
\hline Hyperlipidaemia & 0.92 & 1.2 & 0.77 & $2.5(0.3,5.4)$ & 0.45 \\
\hline Age & 0.04 & 0.05 & 0.75 & $1.0(0.9,1.1)$ & 0.11 \\
\hline HBA1C & 0.43 & 0.27 & 1.59 & $1.5(0.9,2.7)$ & 0.17 \\
\hline Albumin & 0.21 & 0.15 & 1.39 & $1.2(0.9,1.7)$ & \\
\hline
\end{tabular}


Table 7

Glycaemic pattern and variability comparing haemodialysis and non-haemodialysis day

\begin{tabular}{|c|c|c|c|}
\hline Study & $\begin{array}{l}\text { Population and } \\
\text { methods }\end{array}$ & Glycaemic profile pattern and GV & Conclusion \\
\hline $\begin{array}{l}\text { Abe et al. } \\
(2007)[20]\end{array}$ & $\begin{array}{l}\text { - Mean HbA1c: } 8.1 \pm \\
1.2 \% \text { (Poor control) } \\
\text { vs. } 5.8 \pm 0.62 \% \text { (good } \\
\text { control) } \\
\text { - Method: Plasma } \\
\text { blood glucose } \\
\text { - GV indices: Mean }\end{array}$ & $\begin{array}{l}\text { Glycaemic profile and variability } \\
\text { - Plasma blood glucose decreases significantly } \\
\text { between poor and good control during initial } \\
\text { haemodialysis period as compared to } 2 \mathrm{hr} \text { and } 4 \mathrm{hr} \text { in } \\
\text { haemodialysis. } \\
\text { - Poor control group: Hyperglycaemia appeared post } \\
\text { haemodialysis due to decrease in insulin. } \\
\text { - Poor control group: significant changes in plasma } \\
\text { glucose during haemodialysis and non- } \\
\text { haemodialysis day. }\end{array}$ & $\begin{array}{l}\text { - Hyperglycaemic observed } \\
\text { post haemodialysis. } \\
\text { - Fluctuations more } \\
\text { pronounced during } \\
\text { haemodialysis vs non- } \\
\text { haemodialysis day (poor } \\
\text { control) }\end{array}$ \\
\hline $\begin{array}{l}\text { Kazempour- } \\
\text { Ardenilli et } \\
\text { al. (2009) } \\
{[22]}\end{array}$ & $\begin{array}{l}\cdot \mathrm{n}=17 \text { patients (DM- } \\
\text { ESKD) } \\
\text { - Mean HbA1c: } 6.9 \pm \\
1.2 \% \\
\text { - Method: CGM } \\
\text { - GV indices: Mean, } \\
\text { SD, AUC }\end{array}$ & $\begin{array}{l}\text { Glycaemic profile and variability } \\
\text { HD vs. NHD } \\
\text { - Mean (SD) blood glucose: } 9.8 \pm 3.8 \mathrm{mmol} / \mathrm{L} \text { vs. } 12.6 \\
\pm 5.6 \mathrm{mmol} / \mathrm{L} \\
\text { • } 24 \mathrm{hr} \text { AUC: } 4694 \pm 1988 \mathrm{mmol} .3 \mathrm{~min}^{-1} \text { vs. } 5932.1 \pm \\
2673.6 \mathrm{mmol} .3 \mathrm{~min}^{-1} \\
\text { - Hypoglycaemia: } 3 \text { (17\%) had asymptomatic } \\
\text { hypoglycaemia during first } 24 \mathrm{hrs}\end{array}$ & $\begin{array}{l}\text { - Glucose values are } \\
\text { significantly lower on HD as } \\
\text { compared to non- } \\
\text { haemodialysis day. }\end{array}$ \\
\hline $\begin{array}{l}\text { Mirani et al. } \\
(2010)[23]\end{array}$ & $\begin{array}{l}\cdot \mathrm{n}=12 \text { patients (DM- } \\
\text { ESKD) } \\
\text { - Mean HbA1c: } 7.4 \pm \\
1.1 \% \\
\text { - Method: CGM } \\
\text { - GV indices: Mean, } \\
\text { SD and MAGE }\end{array}$ & $\begin{array}{l}\text { Glycaemic profile and variability } \\
\text { Haemodialysis vs. Non-haemodialysis day } \\
\text { • Mean (SD) blood glucose: } 10.32 \pm 2.7 \mathrm{mmol} / \mathrm{L} \text { vs. } \\
8.5 \pm 1.4 \mathrm{mmol} / \mathrm{L} \\
\cdot \text { SD: } 3.16 \pm 1.7 \mathrm{mmol} / \mathrm{L} \text { vs. } 1.9 \pm 0.6 \mathrm{mmol} / \mathrm{L} \\
\cdot \text { MAGE: } 4.16 \pm 1.2 \mathrm{mmol} / \mathrm{L} \text { vs. } 3.18 \pm 0.6 \mathrm{mmol} / \mathrm{L} \\
\text { • Hypoglycaemia: } 2 \text { (11\%) had asymptomatic } \\
\text { occurring } 6 \text { hrs post dialysis }\end{array}$ & $\begin{array}{l}\text { - Haemodialysis day had } \\
\text { increase GV as compared to } \\
\text { non-haemodialysis day }\end{array}$ \\
\hline $\begin{array}{l}\text { Jung et al. } \\
(2010)[24]\end{array}$ & $\begin{array}{l}\cdot \mathrm{n}=9 \text { patients (DM- } \\
\text { ESKD) } \\
\text { - Mean HbA1c: } 8.6 \pm \\
1.2 \% \\
\text { - Method: CGM } \\
\text { - GV indices: Mean, } \\
\text { SD, AUC and MAGE. }\end{array}$ & $\begin{array}{l}\text { Glycaemic profile and variability } \\
\text { haemodialysis vs non-haemodialysis day } \\
\text { - No difference MAGE between haemodialysis and } \\
\text { non-haemodialysis day. } \\
\text { - More pronounced hypoglycaemia on haemodialysis } \\
\text { day. }\end{array}$ & $\begin{array}{l}\text { - GV not affected by } \\
\text { haemodialysis day. }\end{array}$ \\
\hline
\end{tabular}

\section{Discussion}

Dysglycemia in diabetes mellitus can be depicted as the glycemic triumvirate with its three main components, i.e., sustained chronic hyperglycemia, GV, and hypoglycemic episodes where each component appears as a link in a chain for the development and progression of diabetes-related complication more importantly cardiovascular outcomes [17]. Previous studies had shown that not only the average $\mathrm{HbA} 1 \mathrm{c}$ represents independent risk factors for diabetes complications, but also the short-term daily glycemic variation $[18,19]$. Furthermore, haemodialysis per se is another independent risk factor for GV [20]. Hence, it is paramount important to evaluate the GV among ESKD patients as they are more vulnerable to cardiovascular complications. 
GV defined as by swings in blood glucose level that occur throughout the day, including hypoglycemic periods, post-prandial increases, and other blood glucose fluctuations that occur at the same time on a different day [21]. In our study, GV among ESKD on haemodialysis was generally acceptable, where up to $80 \%$ and $90 \%$ of patients achieved the desired target GV during haemodialysis and non-haemodialysis day, respectively. We observed marked GV differences between our DM-ESKD with up to $33 \%$ experienced high GV compared to NDM-ESKD (up to 12\%) during haemodialysis day, which persists to non-haemodialysis day (Figs. 1 \& 2).

Interestingly, despite not being diabetic, a small percentage of NDM-ESKD experienced high GV during haemodialysis day with none of them showed high GV during non-haemodialysis day. This observation supports the notion that haemodialysis is an independent risk factor for GV even among the NDM-ESKD. Our study is in accordance with other studies (Table 8), which shows haemodialysis worsened glycemic control, and diabetic patients had larger GV as compared to non-diabetic patients [13, 20, 22-25]. 
Table 8

Factors associated with glycaemic variability in diabetic population (DM: diabetes mellitus, GV: glycaemic variability, SMBG: Selfmonitoring blood glucose, CGM: continuous glucose monitoring, SD: standard deviation, CoV: co-efficient variant, MAGE: mean amplitude glucose excursion, MAG: mean absolute glucose, CONGA: continuous overall net glycaemic action, MODD: mean of daily difference, OHA: oral hypoglycaemic agent, GA: glycated albumin, DPP4-i: dipeptidyl-peptidase-4-inhibitor.

\begin{tabular}{|c|c|c|c|}
\hline Study & Population and objectives & $\begin{array}{l}\text { Methods of } \\
\text { assessing } \\
\text { GV }\end{array}$ & Conclusion \\
\hline \multirow{4}{*}{$\begin{array}{l}\text { Kohnert et al. } \\
\text { (2007)[29] }\end{array}$} & • Type 2 DM: 63 pts. & \multirow{2}{*}{$\begin{array}{l}\text { - Cross- } \\
\text { sectional } \\
\text { study }\end{array}$} & \multirow{4}{*}{$\begin{array}{l}\text { - HbA1c was related to chronic } \\
\text { hyperglycaemia and not GV. }\end{array}$} \\
\hline & - Objective: Investigate the association with & & \\
\hline & & $\begin{array}{l}\text { - GV indices: } \\
\text { SD, MAGE }\end{array}$ & \\
\hline & & $\begin{array}{l}\text { - Method: } \\
\text { CGM }\end{array}$ & \\
\hline \multirow{2}{*}{$\begin{array}{l}\text { Borg et al. } \\
(2010)[30]\end{array}$} & • Type 1 DM: 268 pts. & \multirow{2}{*}{$\begin{array}{l}\text { - Cross- } \\
\text { sectional } \\
\text { study }\end{array}$} & - GV weak correlation with $\mathrm{HbA} 1 \mathrm{c}$ \\
\hline & • Type 2 DM: 159 pts. & & \multirow{3}{*}{$\begin{array}{l}\text { - HbA1c related to hyperglycaemia indicator } \\
\text { (pre-prandial > than postprandial) }\end{array}$} \\
\hline \multirow{2}{*}{$\begin{array}{l}\text { A1c-derived } \\
\text { Average Blood } \\
\text { Glucose (ADAG) } \\
\text { study }\end{array}$} & - Objective: Examine the relationship between & $\begin{array}{l}\text { - GV indices: } \\
\text { SD, MAGE }\end{array}$ & \\
\hline & & $\begin{array}{l}\text { - Method: } \\
\text { SMBG and } \\
\text { CGM }\end{array}$ & \\
\hline \multirow{4}{*}{$\begin{array}{l}\text { Greven et al. } \\
(2010)[31]\end{array}$} & • Type 1 DM: 166 pts. & \multirow{2}{*}{$\begin{array}{l}\text { - Cross- } \\
\text { sectional } \\
\text { study }\end{array}$} & $\cdot$ T1DM > T2DM in terms of GV \\
\hline & • Type 2 DM: 58 pts. & & \multirow[t]{3}{*}{ • Higher GV in longer insulin therapy } \\
\hline & $\begin{array}{l}\text { - Objective: Investigate different GV } \\
\text { parameters in inadequately controlled T1DM } \\
\text { and T2DM on insulin }\end{array}$ & $\begin{array}{l}\text { - GV indices: } \\
\text { SD, CONGA, } \\
\text { CoV, MDD }\end{array}$ & \\
\hline & & $\begin{array}{l}\text { - Method: } \\
\text { CGM and } \\
\text { SMBG }\end{array}$ & \\
\hline \multirow{4}{*}{$\begin{array}{l}\text { Kuenen et al. } \\
(2011)[32]\end{array}$} & - Type 1 DM: 268 pts & \multirow{2}{*}{$\begin{array}{l}\text { - Cross- } \\
\text { sectional } \\
\text { study }\end{array}$} & \multirow{4}{*}{$\begin{array}{l}\cdot \text { GV indices correlate with higher } \mathrm{HbA} 1 \mathrm{c} \text { in } \\
\text { T1DM } \\
\text { - No association with T2DM }\end{array}$} \\
\hline & • Type 2 DM: 159 pts & & \\
\hline & $\begin{array}{l}\text { - Objective: assessing GV indices with mean- } \\
\text { blood glucose/HbA1c }\end{array}$ & $\begin{array}{l}\cdot \text { GV indices: } \\
\text { SD and } \\
\text { CONGA }\end{array}$ & \\
\hline & & $\begin{array}{l}\text { - Method: } \\
\text { CGM and } \\
\text { SMBG }\end{array}$ & \\
\hline \multirow{4}{*}{$\begin{array}{l}\text { Sartore et al. } \\
(2012)[33]\end{array}$} & • Type 1 DM: 35 pts. & \multirow{2}{*}{$\begin{array}{l}\text { - Cross- } \\
\text { sectional } \\
\text { study }\end{array}$} & - Higher GV in T1DM and long-standing DM \\
\hline & $\begin{array}{l}\text { - Type } 2 \text { DM: } 17 \text { pts. On BB and } 16 \text { pts. On } \\
\text { OHA/basal insulin }\end{array}$ & & \multirow[t]{3}{*}{$\begin{array}{l}\text { - No correlation with insulin treatment and } \\
\text { HbA1c }\end{array}$} \\
\hline & $\begin{array}{l}\text { - Objective: Investigate the association with } \\
\mathrm{HbA} 1 \mathrm{c} \text { and GV }\end{array}$ & $\begin{array}{l}\text { SD and } \\
\text { CONGA }\end{array}$ & \\
\hline & & $\begin{array}{l}\text { - Method: } \\
\text { CGM }\end{array}$ & \\
\hline
\end{tabular}




\begin{tabular}{|c|c|c|c|}
\hline Study & Population and objectives & $\begin{array}{l}\text { Methods of } \\
\text { assessing } \\
\text { GV }\end{array}$ & Conclusion \\
\hline \multirow{4}{*}{$\begin{array}{l}\text { Fang et al. } \\
(2012)[34]\end{array}$} & • Type 2 DM: 291 pts (elderly male) & \multirow{2}{*}{$\begin{array}{l}\text { - Cross- } \\
\text { sectional } \\
\text { study }\end{array}$} & \multirow{4}{*}{$\begin{array}{l}\text { - GV correlate with HbA1c i.e. higher GV in } \\
\text { higher HbA1c }\end{array}$} \\
\hline & - Objective: Influence of GV on HbA1c & & \\
\hline & & $\begin{array}{l}- \text { GV indices: } \\
\text { SD and } \\
\text { MAGE }\end{array}$ & \\
\hline & & $\begin{array}{l}\text { - Method: } \\
\text { CGM }\end{array}$ & \\
\hline \multirow{4}{*}{$\begin{array}{l}\text { Tanaka et al. } \\
(2014)[35]\end{array}$} & - Type 1 DM: 20 pts & \multirow{2}{*}{$\begin{array}{l}\text { - Cross- } \\
\text { sectional } \\
\text { study }\end{array}$} & $\cdot \mathrm{T} 1 \mathrm{DM}>\mathrm{T} 2 \mathrm{DM}$ in terms of $\mathrm{GV}$ \\
\hline & • Type 2 DM: 88 pts & & \multirow{3}{*}{$\begin{array}{l}\text { - GV association: older age, longer duration } \\
\text { diabetes, GA/HbA1c and beta-cell function } \\
\text { (c-peptide level) }\end{array}$} \\
\hline & $\begin{array}{l}\text { - Objective: Assessing the determinants of } \\
\text { GV in Japanese patients with diabetes }\end{array}$ & $\begin{array}{l}- \text { GV indices: } \\
\text { SD and } \\
\text { MAGE }\end{array}$ & \\
\hline & & $\begin{array}{l}\text { - Method: } \\
\text { CGM }\end{array}$ & \\
\hline \multirow{3}{*}{$\begin{array}{l}\text { Juarez et al. } \\
(2012)[36]\end{array}$} & • Type 2 DM: 2970 pts. & \multirow{2}{*}{$\begin{array}{l}\text { Retrospective } \\
\text { - GV indices: } \\
\text { HbA1c }\end{array}$} & \multirow{3}{*}{$\begin{array}{l}\text { - Factors associated with poor glycaemic } \\
\text { control and wide glycaemic variability: Age, } \\
\text { longer duration of DM, multi-pharmacy }\end{array}$} \\
\hline & $\begin{array}{l}\text { - Objective: Identify factors associated with } \\
\text { sustained poor glycaemic control and } \\
\text { glycaemic variability }\end{array}$ & & \\
\hline & & $\begin{array}{l}\text { - Method: } \\
\text { HbA1c serial } \\
\text { assessment }\end{array}$ & \\
\hline \multirow{4}{*}{$\begin{array}{l}\text { Yoo et al. (2015) } \\
\text { [37] }\end{array}$} & • Type 2 DM: 209 pts. & \multirow{2}{*}{$\begin{array}{l}\text { - Cross- } \\
\text { sectional } \\
\text { study }\end{array}$} & \multirow{4}{*}{$\begin{array}{l}\text { - High GV associated with sulfonylureas } \\
\text { used } \\
\text { - Lower in DPP4-i }\end{array}$} \\
\hline & - Objective: Identify factors associated with & & \\
\hline & & $\begin{array}{l}\text { - GV indices: } \\
\text { SD and MAG }\end{array}$ & \\
\hline & & $\begin{array}{l}\text { - Method: } \\
\text { SMBG }\end{array}$ & \\
\hline \multirow{4}{*}{$\begin{array}{l}\text { Mori et al. } \\
(2017)[38]\end{array}$} & • Type 2 DM: 45 pts. & \multirow{2}{*}{$\begin{array}{l}\text { - Cross- } \\
\text { sectional } \\
\text { study }\end{array}$} & \multirow{4}{*}{$\begin{array}{l}\text { - High GV associated with HbA1c, GA level, } \\
\text { female, high insulin dose. }\end{array}$} \\
\hline & $\begin{array}{l}\text { - Objective: Identify factors influencing GV in } \\
\text { diabetic patients receiving insulin therapy }\end{array}$ & & \\
\hline & & $\begin{array}{l}- \text { GV indices: } \\
\text { MODD }\end{array}$ & \\
\hline & & $\begin{array}{l}\text { - Method: } \\
\text { CGM }\end{array}$ & \\
\hline
\end{tabular}

Although we observed a higher GV among DM-ESKD with HbA1c 8-10\%, the sole use of HbA1c in ESKD is limited by several factors, e.g., anaemia, uremia, acidosis, and malnutrition [26]. In the general population, there is a linear relationship between $\mathrm{HbA} 1 \mathrm{c}$ and mean blood glucose with R2 more than 0.80 , which makes HbA1c as an excellent surrogate marker for glycaemic control [27]. In our study, the relationships between mean blood glucose and $\mathrm{HbA} 1 \mathrm{c}$ were moderate, with $\mathrm{R}^{2}=0.59$ [12]. Our result was similar to other bigger studies among haemodialysis patients, where the relationship $\left(R^{2}\right)$ is not more than 0.50 [28]. Therefore, knowledge of factors associated with GV is essential as it allows health professionals to provide targeted interventions to patients with a higher risk of diabetic complications. Currently, many studies that investigate factors affecting GV were done amongst diabetic patients with normal renal function. Moreover, results from these studies varied among each other with small sample size and different indexes of measuring GV (Table 9) [29-38].

In our study, we report that GV, as reported by SMBG, is higher in the older age group, DM-ESKD and patients with hyperlipidemia. Blood parameters associated with high GV was HbA1c, ferritin level, lipid profile and albumin. HbA1c level and its association with 
GV and mean blood glucose among patients had been heavily investigated previously with inconsistent results. In our study, HbA1c was associated with higher GV, especially in the group with $\mathrm{HbA} 1 \mathrm{c}$ between 8-10\%. Currently, the literature on the association of $\mathrm{HbA1c}$ with GV has varied results with studies showed that $\mathrm{HbA1c}$ had a weak correlation with $\mathrm{GV}$ but had a significant relation with chronic hyperglycemia and average blood glucose $[29,30,32,33]$ Conversely, recent studies among the Asian population, showed similar findings with our study where HbA1c correlates well with GV indices [34,35]. However, most of these studies include only normal renal function patients where $\mathrm{HbA1c}$ were more reliable as a surrogate marker and would not be affected with anaemia which is commonly seen among ESKD patients.

GV may be related to pancreatic beta cells dysfunction and insulin resistance, which may occur part of the aging process and duration of illness. In our study, older age group was associated with higher GV which coincide with a previous study done among the Asian population that shown association among the older age group, longer duration of diabetes and low c-peptide [34, 35]. Types of medication also may reflect the process of pancreatic beta-cell dysfunction. Although no association between GV with types of medication was found in this study, previous studies did show an association between insulin and sulfonylureas (insulin secretagogues) treatment with higher GV $[31,37,38]$. In T2DM, beta-cell dysfunction plays a significant role in explaining dysglycaemia, where insufficient insulin secretion for accurate regulation may lead to glucose-related metabolic disorders, which might expose patients to increase GV and sustained hyperglycemia [39-41]. Furthermore, aging per-say has a significant influence on pancreatic $B$ cells function as their functions decline with age with limited capability to regenerate $[42,43]$.

Hyperlipidaemia is recognized as a risk factor for IHD and coronary mortality and was associated with high GV in our study [44, 45]. High-sensitive C-reactive protein (hs-CRP) were used to prognosticate cardiovascular risk in our population, and although it is not a significant association with GV, we found that both DM-ESKD and NDM-ESKD has a higher level of hs-CRP with mean of $8.91 \mathrm{mg} / \mathrm{L}$ and $7.03 \mathrm{mg} / \mathrm{L}$ respectively [12]. GV may further increase cardiovascular risk by propagating oxidative stress, which leads to endothelial dysfunction and angiopathies [46]. In our study, higher ferritin, although non-specific inflammatory markers, were seen higher in the target GV group compared to the high GV group. Nonetheless, patients with high GV also demonstrate high ferritin level with a mean value $554.1 \mathrm{ug} / \mathrm{L}$. A study has shown that chronic inflammation is a risk factor for accelerated atherosclerosis in ESKD patients with markers such as ferritin and quantitative C-reactive protein level can be used to predict the cardiovascular outcome [47]. A study done by using a more specific marker for oxidative stress, i.e., N, N-diethyl paraphenylenediamine, showed that high GV associated with high oxidative stress [48]. In our cohort, the albumin level was lower among DM-ESKD as compared to NDM-ESKD and was associated with high GV. A 10-year cohort study evaluated serum albumin, C-reactive protein, and carotid atherosclerosis as predictors of 10-year mortality in haemodialysis patients shows that serum albumin concentration was a better predictor of mortality [50]. Hence, targeting chronic inflammatory state and improving nutrition could be emerging future research in haemodialysis patients to minimize the associated GV.

Our study has limitations; firstly, the cross-sectional design of the study, and one-off blood sugar monitoring during haemodialysis and non-haemodialysis day SMBG instead of the continuous glucose monitoring system (CGMS) in assessing glycaemic variability. CGMS may be the preferable systems in assessing GV as SMBG can miss specific peaks and nadir in glucose values where the mean amplitude of glycaemic excursion (MAGE) remains the preferable index for GV analysis [51, 52]. However, it is challenging to perform CGM in daily practice, given discomfort, costly, and the need for calibration compared to the SMBG. The practical aspect of SMBG in terms of easy availability, monitoring, and interpretation coupled with relatively cheaper cost makes SMBG be a preferred method in our population. Furthermore, studies had shown that SMBG correlates strongly with GV indices obtained from CGMS [52, 53]. We did not limit or measure the dietary intake of the patients during the study period, which makes dietary intake as a cofounding factor in the glycaemic profile of the patients. Some previous studies will restrict dietary intake or will ask the patient to fast during HD to avoid dietary intake to influence the reading. However, by doing that, it might not represent the normal day-to-day glucose fluctuations of the patients. Moreover, by allowing usual dietary intake, it would be more practical, reflect real-life data, and subsequently may allow alteration in management.

\section{Conclusion}

ESKD patient experienced significant GV during haemodialysis and non-haemodialysis day with a more pronounced effect seen among DM-ESKD. GV predisposed to diabetic complications in particular cardiovascular outcomes, and in our study showed that older age group, DM-ESKD, hyperlipidemia, high HbA1c, ferritin and albumin were associated with high GV. These factors correlate to the progression of the illness, beta-cells dysfunctions and chronic malnutrition-inflammatory state seen among ESKD patients.

Page $16 / 21$ 
Regular glucose monitoring in particular during haemodialysis day may be beneficial in these group of patients to optimize management and to reduce diabetic-related complications.

\section{Abbreviations}

ALP = Alkaline phosphatase

$\mathrm{BMI}=$ Body mass index

CGMS = continuous glucose monitoring system

DM-ESKD = Diabetic End-Stage-Kidney-Disease

ESKD $=$ End-Stage-Kidney-Disease

GV = Glycemic variability

HbA1c $=$ Hemoglobin A1c

$\mathrm{HB}=$ hemoglobin

HDD = Haemodialysis day

$\mathrm{HDL}=$ High density lipoprotein

Hs-CRP = Highly sensitive C-reactive protein

$I H D=$ Ischemic heart disease

$\mathrm{iPTH}=$ Intact parathyroid hormone

LDL = low density lipoprotein;

MAGE = Mean Amplitude Of Glycaemic Excursion

NDM-ESKD = Non-diabetic End-Stage-Kidney-Disease

NHDD $=$ Non-Haemodialysis day

SD = Standard deviation

SMBG = Self-monitoring blood glucose

$\mathrm{TG}=$ triglycerides

T2DM = Type 2 Diabetes Mellitus

$\% \mathrm{CV}=$ percentage co-efficient variant

\section{Declarations}

\section{ETHICS APPROVAL AND CONSENT TO PARTICIPATE}

This study was approved by University Putra Malaysia Ethics Committee involving human (UPM/TNCPI/RMC/1.4.18.2/JKEUPM), and was carried out in accordance with the principles of the Declaration of Helsinki.

\section{CONSENT FOR PUBLICATION}

Not applicable. 


\section{COMPETING INTERESTS}

No conflict of interest.

\section{FUNDING}

This study was supported by Putra Grant University Putra Malaysia (UPM/700-1/3/Geran Putra). University Putra Malaysia is a research university and the study was part of institutional research program.

\section{AUTHORS' CONTRIBUTION}

Conceptualization: NFZ, NAK, AHKYK; Data Curation: NFZ, AHKYK, AHK, Funding: NFZ, AHKYK; Formal Analysis: AHKYK, MAZA; Investigation and Methodology: NFZ, AHKYK, MAZA; Sofware: MAZA; Supervision: NFZ, NAK; Writing original draft: NFZ, AHKYK, MAZA; Writing - review \& editing: NFZ, AHKYK, MAZA,

\section{All authors have read and approved the manuscript.}

\section{ACKNOWLEDGEMENT}

The authors would like to thank all the staff and patients from Pusat Perubatan Dialysis Bangi, Dengkil, Semenyih, Farah Mahami Dialysis Center, and Bangi Dialysis Centre.

\section{AVAILABILITY OF DATA AND MATERIAL}

The datasets used during the current study are available from the corresponding author on reasonable request.

\section{References}

1. Excerpts from the US Renal Data System 2009 Annual Data Report Collins AJ, Foley RN, Herzog C, Chavers BM, Gilbertson D, Ishani A, et al. Excerpts from the US Renal Data System 2009 Annual Data Report. Am J Kidney Dis. 2010;55 1 Suppl 1:1.

2. Couser WG, Remuzzi G, Mendis S, Tonelli M. The contribution of chronic kidney disease to the global burden of major noncommunicable diseases. Kidney Int. 2011;80:1258-70.

3. Wong H, Goh B. Twenty Third Report of The Malaysian Dialysis and Transplant 2015. Kuala Lumpur 2017; 2017. http://www.msn.org.my.

4. Kalantar-Zadeh K, Kopple JD, Regidor DL, Jing J, Shinaberger CS, Aronovitz J, et al. A1C and survival in maintenance haemodialysis patients. Diabetes Care. 2007;30:1049-55.

5. Rhee CM, Leung AM, Kovesdy CP, Lynch KE, Brent GA, Kalantar-Zadeh K. Updates on the management of diabetes in dialysis patients. Semin Dial. 2014;27:135-45.

6. Patel A, MacMahon S, Chalmers J, Neal B, Billot L, Woodward M, et al. Intensive blood glucose control and vascular outcomes in patients with type 2 diabetes. N Engl J Med. 2008;358:2560-72.

7. Duckworth W, Abraira C, Moritz T, Reda D, Emanuele N, Reaven PD, et al. Glucose control and vascular complications in veterans with type 2 diabetes. N Engl J Med. 2009;360:129-39.

8. ACCORD Study Group. Effects of Intensive Glucose Lowering in Type 2 Diabetes. N Engl J Med. 2008;358:2545-59.

9. Nalysnyk L, Hernandez-Medina M, Krishnarajah G. Glycaemic variability and complications in patients with diabetes mellitus: evidence from a systematic review of the literature. Diabetes Obes Metab. 2010;12:288-98.

10. Ramirez SPB, McCullough KP, Thumma JR, Nelson RG, Morgenstern H, Gillespie BW, et al. Hemoglobin A1c levels and mortality in the diabetic haemodialysis population: Findings from the Dialysis Outcomes and Practice Patterns Study (DOPPS). Diabetes Care. 2012;35:2527-32.

11. Ricks J, Molnar MZ, Kovesdy CP, Shah A, Nissenson AR, Williams M, et al. Glycemic control and cardiovascular mortality in haemodialysis patients with diabetes: A 6-year cohort study. Diabetes. 2012;61:708-15. 
12. Khan AHKY, Zakaria NF, Abidin MAZ, Lim CTS, Kamaruddin NA. Glycemic Patterns and Factors Associated with PostHaemodialysis Hyperglycemia among End-Stage Renal Disease Patients undergoing Maintenance Haemodialysis. J ASEAN Fed Endocr Soc. 2020;35:68-76. doi:10.15605/jafes.035.01.12.

13. Jin YP, Su XF, Yin GP, Xu XH, Lou JZ, Chen JJ, et al. Blood glucose fluctuations in haemodialysis patients with end stage diabetic nephropathy. J Diabetes Complications. 2015;29:395-9.

14. Faul F, Erdfelder E, Lang AG, Buchner A. G*Power 3: A flexible statistical power analysis program for the social, behavioral, and biomedical sciences. Behav Res Methods. 2007;39:175-91.

15. Hirsch IB. Glycemic variability and diabetes complications: Does it matter? Of course it does! Diabetes Care. 2015;38:1610-4.

16. Monnier L, Colette C, Wojtusciszyn A, Dejager S, Renard E, Molinari N, et al. Toward defining the threshold between low and high glucose variability in diabetes. Diabetes Care. 2017;40:832-8.

17. Monnier L, Colette C, Owens D. The glycemic triumvirate and diabetic complications: Is the whole greater than the sum of its component parts? Diabetes Res Clin Pract. 2012;95:303-11.

18. Home P. Contributions of basal and post-prandial hyperglycaemia to micro- and macrovascular complications in people with type 2 diabetes. Curr Med Res Opin. 2005;21:989-98.

19. Colette C, Monnier L. Acute glucose fluctuations and chronic sustained hyperglycemia as risk factors for cardiovascular diseases in patients with type 2 diabetes. Horm Metab Res. 2007;39:683-6.

20. Abe M, Kaizu K, Matsumoto K. Plasma insulin is removed by haemodialysis: Evaluation of the relation between plasma insulin and glucose by using a dialysate with or without glucose. Ther Apher Dial. 2007;11:280-7.

21. Suh S, Kim JH. Glycemic variability: How do we measure it and why is it important? Diabetes Metab J. 2015;39:273-82.

22. Kazempour-Ardebili S, Lecamwasam VL, Dassanyake T, Frankel AH, Tam FWK, Dornhorst A, et al. Assessing glycemic control in maintenance haemodialysis patients with type 2 diabetes. Diabetes Care. 2009;32:1137-42.

23. Mirani M, Berra C, Finazzi S, Calvetta A, Radaelli MG, Favareto F, et al. Inter-day glycemic variability assessed by continuous glucose monitoring in insulin-treated type 2 diabetes patients on haemodialysis. Diabetes Technol Ther. 2010;12:749-53.

24. Jung HS, Kim HII, Kim MJ, Yoon JW, Ahn HY, Cho YM, et al. Analysis of haemodialysis-associated hypoglycemia in patients with type 2 diabetes using a continuous glucose monitoring system. Diabetes Technol Ther. 2010;12:801-7.

25. Gai M, Merlo I, Dellepiane S, Cantaluppi V, Leonardi G, Fop F, et al. Glycemic pattern in diabetic patients on haemodialysis: Continuous Glucose Monitoring (CGM) analysis. Blood Purif. 2014;38:68-73.

26. Coelho S. What is the Role of HbA1c in Diabetic Haemodialysis Patients? Semin Dial. 2016;29:19-23.

27. Nathan DM, Kuenen J, Borg R, Zheng H, Schoenfeld D, Heine RJ. Translating the A1C assay into estimated average glucose values. Diabetes Care. 2008;31:1473-8.

28. Hoshino J, Mehrotra R, Rhee CM, Yamagata K, Ubara Y, Takaichi K, et al. Using hemoglobin A1c to derive mean blood glucose in peritoneal dialysis patients. Am J Nephrol. 2013;37:413-20.

29. Kohnert KD, Augstein P, Heinke P, Zander E, Peterson K, Freyse EJ, et al. Chronic hyperglycemia but not glucose variability determines HbA1c levels in well-controlled patients with type 2 diabetes. Diabetes Res Clin Pract. 2007;77:420-6.

30. Borg R, Kuenen JC, Carstensen B, Zheng H, Nathan DM, Heine RJ, et al. Associations between features of glucose exposure and A1C: The A1C-Derived Average Glucose (ADAG) study. Diabetes. 2010;59:1585-90.

31. Greven WL, Beulens JWJ, Biesma DH, Faiz S, De Valk HW. Glycemic variability in inadequately controlled type 1 diabetes and type 2 diabetes on intensive insulin therapy: A cross-sectional, observational study. Diabetes Technol Ther. 2010;12:695-9.

32. Kuenen JC, Borg R, Kuik DJ, Zheng H, Schoenfeld D, Diamant M, et al. Does glucose variability influence the relationship between mean plasma glucose and HbA 1c levels in type 1 and type 2 diabetic patients? Diabetes Care. 2011;34:1843-7.

33. Sartore G, Chilelli NC, Burlina S, Stefano P, Di, Piarulli F, Fedele D, et al. The importance of HbA1c and glucose variability in patients with type 1 and type 2 diabetes: Outcome of continuous glucose monitoring (CGM). Acta Diabetol. 2012;49 1 SUPPL.

34. Fang FS, Li ZB, Li CL, Tian H, Li J, Cheng XL. Influence of glycemic variability on the HbA1c level in elderly male patients with type 2 diabetes. Intern Med. 2012;51:3109-13.

35. Tanaka C, Saisho Y, Tanaka K, Kou K, Tanaka M, Meguro S, et al. Factors associated with glycemic variability in Japanese patients with diabetes. Diabetol Int. 2014;5:36-42. 
36. Juarez DT, Sentell T, Tokumaru S, Goo R, Davis JW, Mau MM. Factors associated with poor glycemic control or wide glycemic variability among diabetes patients in Hawaii, 2006-2009. Prev Chronic Dis. 2012;9.

37. Yoo S, Chin SO, Lee SA, Koh G. Factors Associated with Glycemic Variability in Patients with Type 2 Diabetes: Focus on Oral Hypoglycemic Agents and Cardiovascular Risk Factors. Endocrinol Metab (Seoul Korea). 2015;30:352-60.

38. Mori H, Okada Y, Kurozumi A, Narisawa M, Tanaka Y. Factors influencing inter-day glycemic variability in diabetic outpatients receiving insulin therapy. J Diabetes Investig. 2017;8:69-74.

39. DeFronzo RA, Abdul-Ghani MA. Preservation of $\beta$-cell function: The key to diabetes prevention. J Clin Endocrinol Metab. 2011;96:2354-66.

40. Vella A, Zinsmeister AR. Predicting diabetes using measures of $\beta$-cell function. Diabetes. 2012;61:562-3.

41. Ize-Ludlow D, Lightfoot $Y L$, Parker $M$, Xue S, Wasserfall $C$, Haller MJ, et al. Progressive erosion of $\beta$-cell function precedes the onset of hyperglycemia in the NOD mouse model of type 1 diabetes. Diabetes. 2011;60:2086-91.

42. Gu Z, Du Y, Liu Y, Ma L, Li L, Gong Y, et al. Effect of aging on islet beta-cell function and its mechanisms in Wistar rats. Age (Omaha). 2012;34:1393-403.

43. Tschen SI, Dhawan S, Gurlo T, Bhushan A. Age-dependent decline in $\beta$-cell proliferation restricts the capacity of $\beta$-cell regeneration in mice. Diabetes. 2009;58:1312-20.

44. Nelson RH. Hyperlipidemia as a Risk Factor for Cardiovascular Disease. Prim Care - Clin Off Pract. 2013;40:195-211. doi:10.1016/j.pop.2012.11.003.

45. Lacoste L, Lam JYT, Hung J, Letchacovski G, Solymoss CB, Waters D. Hyperlipidemia and Coronary Disease. Circulation. 1995;92:3172-7. doi:10.1161/01.CIR.92.11.3172.

46. Probstfield JL. Design of FLAT-SUGAR: Randomized trial of prandial insulin versus prandial glp-1 receptor agonist together with basal insulin and metformin for high-risk type 2 diabetes. Diabetes Care. 2015;38:1558-66.

47. Zoccali C, Mallamaci F, Tripepi G. Novel Cardiovascular Risk Factors in End-Stage Renal Disease. J Am Soc Nephrol. $2004 ; 151$ SUPPL.

48. Ohara M, Fukui T, Ouchi M, Watanabe K, Suzuki T, Yamamoto S, et al. Relationship between daily and day-to-day glycemic variability and increased oxidative stress in type 2 diabetes. Diabetes Res Clin Pract. 2016;122:62-70.

49. Kovesdy CP, Kalantar-Zadeh K. Why Is Protein-Energy Wasting Associated With Mortality in Chronic Kidney Disease? Semin Nephrol. 2009;29:3-14.

50. Kato A, Takita T, Furuhashi M, Maruyama Y, Hishida A. Comparison of serum albumin, C-reactive protein and carotid atherosclerosis as predictors of 10-year mortality in haemodialysis patients. Hemodial Int. 2010;14:226-32.

51. Service FJ. Glucose variability. Diabetes. 2013;62:1398-404.

52. Rodbard D. Interpretation of continuous glucose monitoring data: Glycemic variability and quality of glycemic control. Diabetes Technol Ther. 2009;11 SUPPL.1.

53. Kohnert K-D, Heinke P, Fritzsche G, Vogt L, Augstein P, Salzsieder E. Evaluation of the Mean Absolute Glucose Change as a Measure of Glycemic Variability Using Continuous Glucose Monitoring Data. Diabetes Technol Ther. 2013;15:448-54.

\section{Figures}


Mean Glucose During Hemodialysis Day Based on GV index (SD)

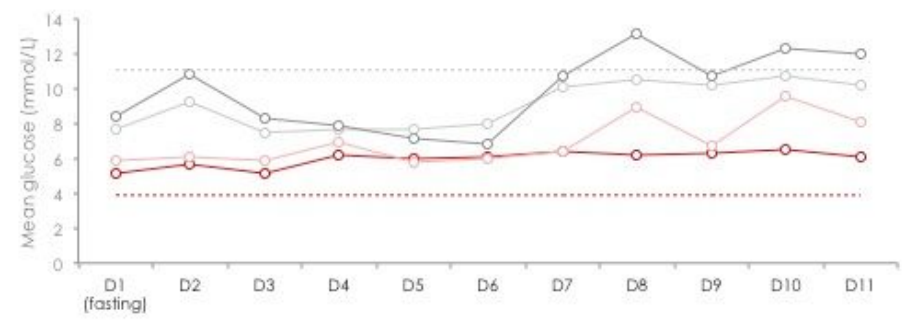

Hemalalysis Day |time)

-O-DM-ESKD (mmol/L) Target SD

$\rightarrow$ NDM-ESKD (mmol/L) Target SD

Hyperglycemia $\longrightarrow-$ DM-ESKD (mmol/L) High SD
$\multimap-$ NDM-ESKD (mmol/L) High SD
$\ldots \ldots$... Hypoglycemia
Mean Glucose During Hemodialysis Day Based on GV index (\%CV)

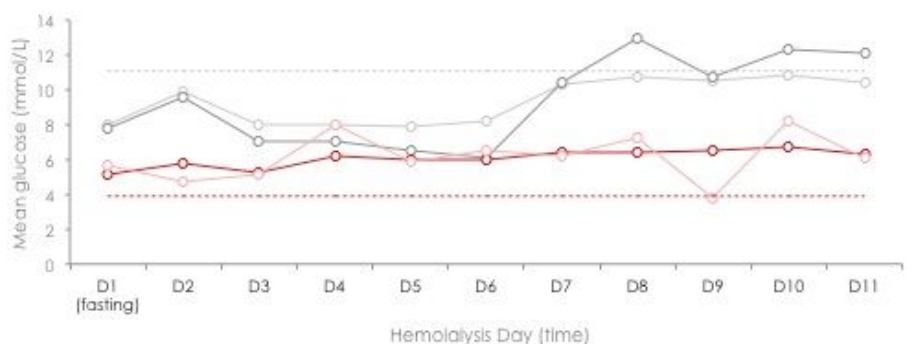

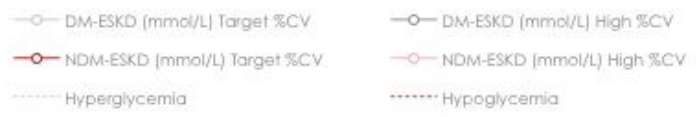

\section{Figure 1}

Glycaemic pattern during haemodialysis day (HD) based on GV indices, i.e., SD and \%CV. Both graphs show that glycaemic fluctuations were more marked among patients with high GV indices and in DM-ESKD. Timing: D1 = Fasting, D2 = Prior haemodialysis, D3 = 1st hour haemodialysis, D4 = 2nd hour haemodialysis, D5 = 3rd hour haemodialysis, D6 $=4$ th hour haemodialysis, D7 $=2$ hours post haemodialysis, before meal, D8 $=2 \mathrm{hrs}$ post-meal, D9 $=$ before dinner, D10 $=2$ hours post-dinner, D11 $=$ before sleep.
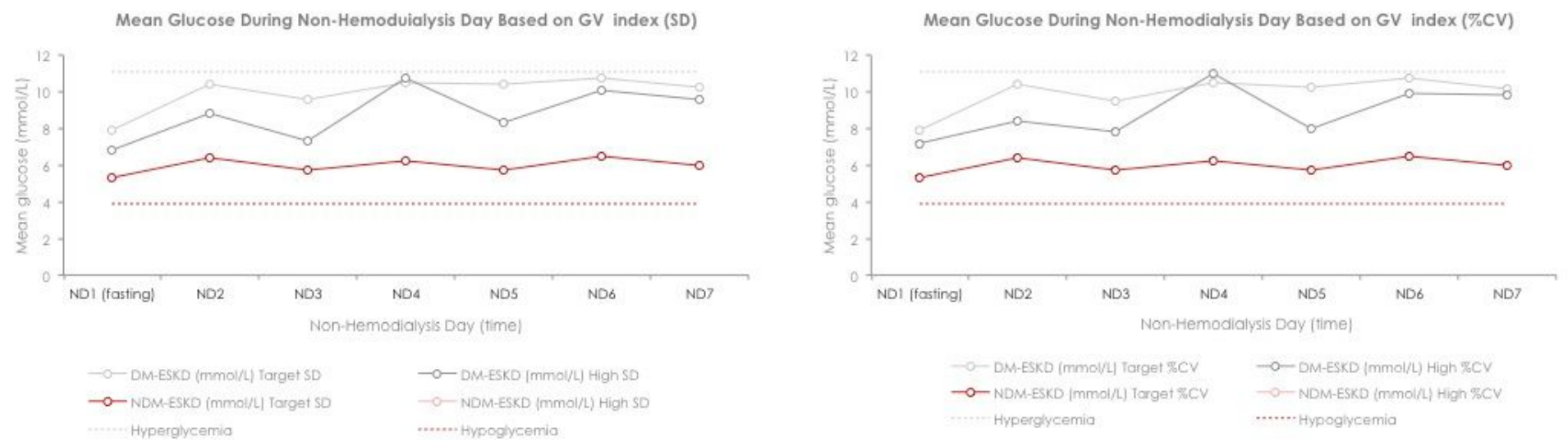

\section{Figure 2}

Glycaemic pattern during non-haemodialysis day (N-HD) based on GV indices, i.e., SD and \%CV. Both graphs show that glycaemic fluctuations were more marked among DM-ESD with high GV indices. No NDM-ESKD had high GV during non-haemodialysis day. Timing: ND1 = Fasting - before breakfast, ND2 $=2$ hours post breakfast, ND3 = before lunch, ND4 $=2$ hours post-lunch, ND5 = before dinner, ND6 = 2 hours post-dinner, ND7 = before sleep.

\section{Supplementary Files}

This is a list of supplementary files associated with this preprint. Click to download.

- questionnaireGV.pdf 